\title{
Impulsive Control on Seasonally Perturbed General Holling Type Two-Prey One-Predator Model
}

\author{
Chandrima Banerjee and Pritha Das \\ Department of Mathematics, Indian Institute of Engineering Science and Technology, Shibpur, Howrah, West Bengal 711103, India \\ Correspondence should be addressed to Chandrima Banerjee; chandrimab05@gmail.com
}

Received 13 April 2016; Revised 10 June 2016; Accepted 16 June 2016

Academic Editor: Zhengqiu Zhang

Copyright ( 92016 C. Banerjee and P. Das. This is an open access article distributed under the Creative Commons Attribution License, which permits unrestricted use, distribution, and reproduction in any medium, provided the original work is properly cited.

\begin{abstract}
We investigate the dynamical behaviors of two-prey one-predator model with general Holling type functional responses. The effect of seasonal perturbation on the model has been discussed analytically as well as numerically. The periodic fluctuation is considered in prey growth rate and the predator mortality rate of the model. The impulsive effects involving biological and chemical control strategy, periodic releasing of natural enemies, and spraying pesticide at different fixed times are introduced in the model with seasonal perturbation. We derive the conditions of stability for impulsive system using Floquet theory, small amplitude perturbation skills. A local asymptotically stable prey (pest) eradicated periodic solution is obtained when the impulsive period is less than some critical value. Numerical simulations of the model with and without seasonal disturbances exhibit different dynamics. Also we simulate numerically the model involving seasonal perturbations without impulse and with impulse. Finally, concluding remarks are given.
\end{abstract}

\section{Introduction}

Functional response plays an important role in dynamics of population ecology. There are several examples in nature where the predator population affects the number of their prey populations. When predators are faced with increasing local density of their prey, they often respond by changing their consumption rate. This relationship of an individual predator's rate of food consumption with the prey density was first suggested by Holling [1] as functional response. Functional responses are generally classified into Holling types I, II, III, and IV. Type I is the simplest capture rate which increases in direct proportion to prey density until it abruptly saturates. Similarly in type II, the rate of capture increases with increasing prey density. In contrast to the linear increase of type I, type II approaches saturation gradually at higher prey density. This behavior is better expressed by an asymptotic relationship between per capita feeding rate and prey density which is of the functional form $m N /(1+$ $\widehat{b} N$ ), where $N$ is the prey density. $m$ (per unit time) and $\widehat{b}$ (per unit prey) are positive constants describing the effects of capture rate and handling time on the feeding rate of the predator, respectively. Holling type II functional response is most common type of functional response among arthropod predators. Type III resembles type II in having an upper limit to prey consumption except at low prey density, and the predator's response to prey is depressed. The mathematical expression of type III functional response is of the form $m N^{2} /\left(1+a N^{2}\right)$, where $a>0$ is half-saturation constant. For example, at low prey density, predators switch to alternative prey species, if the focal prey is less accessible due to surplus refuges [1]. Holling types I, II, and III are all monotonically nondescending in the first quadrant [2]. But for some preypredator systems, a nonmonotonic response (Holling IV) occurs because the growth of predator may be inhibited when the prey density reaches a high level [3]. Holling type IV functional response is written as $m N /\left(1+b N+a N^{2}\right)$. This is also known as Monod-Haldane-type functional response [4], where $b>-2 \sqrt{a}$ as the denominator of the expression does not vanish for nonnegative $N$. When $b=0$, a simplified form $m N /\left(1+a N^{2}\right)$ is proposed by Sokol and Howell [5], and some researchers also called it Holling type IV [4, 6]. 
In population dynamics, this functional response describes the phenomenon of group defense which causes a decrease or prevention of predation due to the increased ability of the prey to better defend or disguise themselves when their numbers are large enough. Lone ox can be attacked successfully by wolves in regular basis. While small herds of musk ox grazing in group are attacked by wolves with less success rate. But successful attacks on larger herds are not observed in general [7] (see Tener, 1965).

Kooij and Zegeling [8] and Sugie et al. [9] considered the predator's functional response term of the form $g(x)=$ $x^{n} /\left(c+x^{n}\right)$, where $n$ is a positive constant. $c>0$ is the conversion rate of prey captured by predator. This particular type functional response with $n \geq 1$ was introduced by Kazarinoff and van den Driessche [10]. This is known as general Holling type functional response.

A model for two-prey one-predator ecological system with general Holling type functional responses is discussed here. In this paper, apparent competition between the prey is treated. Only the predator hunting time for each prey depending on the selection and availability of its favorite prey is considered. Most of the models are considered with constant environment which is not ecologically realistic. There are many factors in the environment which are not constant but vary periodically with change in time such as concentration of atmospheric carbon dioxide, climate, and ecological succession. It is natural to identify that seasonality plays an important functional role in changing the behavior of individual population. In the last decades, many scientists [11-15] have studied the interactions between seasonality and internal biological rhythms of simple predator-prey systems. Without seasonal variations the two-species nonlinear autonomous dynamical system has either stable or periodic solution. Seasonality introduces complex dynamical behavior in the system such as the existence of multiple attractors, catastrophes, and chaos [15-19]. In this paper we investigate how two-prey one-predator model with general Holling type functional responses changes its dynamics with change in seasonal parameters like degree of seasonality. The periodic variations in the parameters may not be synchronous due to seasonality. Seasonality parameters with different phases reach their maximum influence at different times [19]. We consider seasonal variation in prey growth rate and predator mortality rate with different phase angles between them. We investigate the boundedness of the model and the condition of uniform persistence.

The effects of impulsive perturbation in population ecology have been widely studied and discussed by number of researchers [20-26]. Impulsive perturbations bring sudden changes in the system. Ecological model with combined effect of impulsive and seasonal perturbation has been investigated by few researchers [27-30]. Pei et al. [20] have considered also two prey and one predator with group defense and Holling type II functional responses and investigated the effect of impulsive perturbation in pest control using pesticides. In this paper, we study general Holling type two prey (pests) and one predator with seasonal disturbances as well as proportional periodic impulsive poisoning (spraying pesticide) for all species and constant periodic releasing, or immigrating, for the predator at different fixed time. We study the local stability of the prey- (pest-) free periodic solution.

The rest of this paper is organized as follows. In Section 2, a detailed description about mathematical formulation of model with seasonal variation is given. Then the model is modified by incorporating impulsive perturbation in it. In Section 3, we discuss various important theorems and lemmas. In Section 4, the boundedness and uniform persistence of the solution of the system are proved. Expression for preyfree periodic solution of the system is obtained in Section 5. We derive the conditions for stability of the prey- (pest-) free periodic solution. In Section 6, results of numerical analysis are presented. In Section 7 the results are discussed thoroughly.

\section{Mathematical Model Formulation}

2.1. Model Formulation without Impulsive Effect. We consider the following deterministic model with general Holling type function responses and seasonally perturbed parameter is described by the following system of differential equations:

$$
\begin{aligned}
\frac{d x(t)}{d t} & =x(t)\left(a_{1}-x(t)\right)-c_{1} \frac{x^{p}(t) z(t)}{1+e_{1} x^{q}(t)}, \\
\frac{d y(t)}{d t} & =y(t)\left(a_{2}-y(t)\right)-c_{2} \frac{y^{s}(t) z(t)}{1+e_{2} y^{r}(t)}, \\
\frac{d z(t)}{d t} & =z(t)\left[\frac{m_{1} x^{p}(t)}{1+e_{1} x^{q}(t)}+\frac{m_{2} y^{s}(t)}{1+e_{2} y^{r}(t)}-d_{1}\right], \\
a_{1} & \equiv a_{1}\left(1+\varepsilon_{1} \sin \omega t\right), \\
a_{2} & \equiv a_{2}\left(1+\varepsilon_{2} \sin (\omega t+\psi)\right), \\
d_{1} & \equiv d_{1}\left(1+\varepsilon_{3} \sin (\omega t+\phi)\right),
\end{aligned}
$$

where $x(t), y(t)$, and $z(t)$ are the biomass of the two prey and the predator at time $t$, respectively. $a_{i}$ s $(i=1,2)$ are intrinsic birth rates of prey and $d_{1}$ is intrinsic mortality rate of predator, $e_{i} s(i=1,2)$ are parameters representing halfsaturation constants, and $m_{i} s(i=1,2)$ are equal to the transformation rates of predator due to predation (rates of converting prey into predator). $c_{i} s(i=1,2)$ are the per capita predation rates of the predator. All parameters are considered as positive constants $p, q, s, r>0$. The parameters $\varepsilon_{i} s(i=$ $1,2,3)$ represent the degree of seasonality where $0 \leq \varepsilon_{i} \leq 1$. $a_{1} \varepsilon_{1}, a_{2} \varepsilon_{2}$, and $d_{1} \varepsilon_{3}$ are the magnitudes of the perturbations in $a_{1}(t), a_{2}(t)$, and $d_{1}(t)$, respectively [18]. $\omega$ is the angular frequency of the fluctuations caused by seasonality. Finally $\phi$ and $\psi$, where $0 \leq \psi \leq 2 \pi$ and also $0 \leq \phi \leq 2 \pi$, can be interpreted as the difference in phase angles between the seasonality of the above three parameters. Clearly, when $\psi=$ 0 which imply that there is in-phase synchronous variation in between the intrinsic growth rates of prey, while $\psi=\pi$, the variation is antiphase synchronous. In this paper, the values of phase angles will be considered $\psi=0, \pi / 2$, and $\pi$ and $\phi=\pi / 2$. 
2.2. Model Formulation with Impulsive Effect. We develop system (1) by introducing a proportional periodic impulsive poisoning (spraying pesticide) for all species and a constant periodic releasing, or immigrating, for the predator at different fixed times as follows:

$$
\begin{aligned}
& \frac{d x(t)}{d t}=x(t)\left(a_{1}\left(1+\varepsilon_{1} \sin \omega t\right)-x(t)\right) \\
& -\frac{c_{1} x^{p}(t) z(t)}{1+e_{1} x^{q}(t)} \\
& \frac{d y(t)}{d t}=y(t)\left(a_{2}\left(1+\varepsilon_{2} \sin (\omega t+\psi)\right)-y(t)\right) \\
& -\frac{c_{2} y^{s}(t) z(t)}{1+e_{2} y^{r}(t)} \\
& \frac{d z(t)}{d t}=z(t)\left[\frac{m_{1} x^{p}(t)}{1+e_{1} x^{q}(t)}+\frac{m_{2} y^{s}(t)}{1+e_{2} y^{r}(t)}\right. \\
& \left.-d_{1}\left(1+\varepsilon_{3} \sin (\omega t+\phi)\right)\right] \\
& t \neq n T, \quad t \neq(n+l-1) T, \\
& t=(n+l-1) T, \\
& t=n T,
\end{aligned}
$$

where $\Delta x(t)=x\left(t^{+}\right)-x(t), \Delta y(t)=y\left(t^{+}\right)-y(t), \Delta z(t)=$ $z\left(t^{+}\right)-z(t)$. Here $x(t), y(t)$, and $z(t)$ are considered as the biomass of the two prey (pest) and the predator (natural enemy) at time $t$, respectively. $0<l<1,0 \leq \delta_{i}<1$ $(i=1,2,3)$ present the fraction of the prey and the predator which die due to the harvesting or pesticides and so forth. $T$ is the period of the impulsive immigration or stock of the predator. $P>0$ is the size of immigration or stock of the predator. In order to get some conditions guaranteeing the system is permanent, $T$ is the period of spaying pesticides (harvesting) and the impulsive immigration or stock of the predator, respectively. We present the fraction of the prey and the predator which die due to the harvesting or pesticides and so forth.

Incorporating $\sin (\omega t)=\sin (\omega t+\psi)=1$ (see [31]) in system (2), we get

$$
\begin{aligned}
& \frac{d x(t)}{d t}=x(t)\left(a_{1}\left(1+\varepsilon_{1}\right)-x(t)\right)-\frac{c_{1} x^{p}(t) z(t)}{1+e_{1} x^{q}(t)}, \\
& \frac{d y(t)}{d t}=y(t)\left(a_{2}\left(1+\varepsilon_{2}\right)-y(t)\right)-\frac{c_{2} y^{s}(t) z(t)}{1+e_{2} y^{r}(t)},
\end{aligned}
$$

$$
\begin{aligned}
& \frac{d z(t)}{d t} \\
& =z(t)\left[\frac{m_{1} x^{p}(t)}{1+e_{1} x^{q}(t)}+\frac{m_{2} y^{s}(t)}{1+e_{2} y^{r}(t)}-d_{1}\left(1+\varepsilon_{3}\right)\right], \\
& t \neq n T, t \neq(n+l-1) T,
\end{aligned}
$$

$\Delta x=-\delta_{1} x(t)$,

$\Delta y=-\delta_{2} y(t)$,

$\Delta z=-\delta_{3} z(t)$,

$$
t=(n+l-1) T
$$

$\Delta x=0$,

$\Delta y=0$,

$\Delta z=P$,

$$
t=n T \text {. }
$$

\section{Preliminaries and Basic Lemmas}

Definition 1. System (1) is uniformly persistent if, for every positive solution $(x(t), y(t), z(t))$ of system (1), there exist positive constants $m_{x}, m_{y}, m_{z}, M_{x}, M_{y}, M_{z}$, and $T>0$ such that $m_{x}<x(t)<M_{x}, m_{y}<y(t)<M_{y}$, and $m_{z}<z(t)<M_{z}$ for $t \geq T$

Lemma 2 (see [32]). If $a>0, b>0$, and $(d \widehat{h}(t) / d t) \geq(\leq$ )$\widehat{h}(t)\left(b-a \widehat{h}^{1 / \alpha}(t)\right)(\alpha>0)$, then for $t \geq 0$ and $\widehat{h}(0)>0$ one has $\widehat{h}(t) \geq(\leq)(b / a)^{1 / \alpha}\left\{1+\left(b \widehat{h}^{-\alpha}(0) / a-1\right) e^{-b \alpha t}\right\}^{-1 / \alpha}$.

Note 1. From Lemma 2 we get

$$
\begin{aligned}
\liminf \widehat{h}(t) & \geq\left\{\frac{b}{a}\right\}^{1 / \alpha} \\
\text { or } \lim \sup \widehat{h}(t) & \leq\left\{\frac{b}{a}\right\}^{1 / \alpha} .
\end{aligned}
$$

Let $R_{+}=[0, \infty), R_{+}^{3}=\left\{X=(x, y, z) \in R^{3} \mid X \geq 0\right\}$. Let $N$ be the set of nonnegative integers and let $f=\left(f_{1}, f_{2}, f_{3}\right)^{T}$ be defined as the map by the right hand of the system of equations (1). Let $V: R_{+} \times R_{+}^{3} \rightarrow R_{+}$; then $V$ belongs to class $V_{0}$ if

(1) $V$ is continuous in $((n-1) T,(n+l-1) T] \times R_{+}^{3} \cup$ $((n+l-1) T, n T] \times R_{+}^{3}$, and for every $X \in R_{+}^{3}, n \in$ $N \lim _{(t, y) \rightarrow\left((n+l-1) T^{+}, X\right)} V(t, y)=V\left((n+l-1) T^{+}, X\right)$ and $\lim _{(t, y) \rightarrow\left(n T^{+}, X\right)} V(t, X)$ exist;

(2) $V$ is locally Lipschitzian in $X$.

Definition 3. Let $V \in V_{0}$; then for $(t, X) \in((n-1) T$, $(n+l-$ $1) T] \times R_{+}^{3}$ and $((n+l-1) T, n T] \times R_{+}^{3}$, the upper right derivative 
of $V(t, X)$ with respect to the impulsive differential system (2) is defined as

$$
\begin{aligned}
& D^{+} V(t, X) \\
& \quad=\lim _{h \rightarrow 0^{+}} \sup \frac{1}{h}[V(t+h, X+h f(t, X))-V(t, X)] .
\end{aligned}
$$

The solution of system (2) is a piecewise continuous function $X: R_{+} \rightarrow R_{+}^{3}, X(t)$ is continuous on $((n-1) T,(n+$ $l-1) T) \cup((n+l-1) T, n T)$, where $n \in N$ and $0<l<1$, and then $X\left((n+l-1) T^{+}\right)=\lim _{(n+l-1) T^{+}} X(t)$ and $X\left(n T^{+}\right)=$ $\lim _{t \rightarrow n T^{+}} X(t)$ exist. Thus these properties guarantee the global existence of $f$ and uniqueness of solution of system (2) $[33,34]$.

Definition 4. System (2) is permanent if there exists a compact $\Omega \subset$ int $R_{+}^{3}$ such that every solution $(x(t), y(t), z(t))$ of system (2) will enter and remain in the region $X$.

The following lemma is evident.

Lemma 5. Let $X(t)$ be a solution of system (2) with $X\left(0^{+}\right) \geq 0$; then $X(t) \geq 0$ for all $t \geq 0$. Also $X(t)>0, t>0$ if $X\left(0^{+}\right)>0$.

We will use a comparison result of impulsive differential inequalities. Suppose that $g: R_{+} \times R_{+} \rightarrow R$ satisfies the following hypotheses.

(H) $g$ is continuous on $((n-1) T,(n+l-1) T] \times R_{+} \cup((n+$ $l-1) T, n T] \times R_{+}$and the limit $\lim _{(t, y) \rightarrow\left(t_{0}, x\right)} g(t, y)=$ $g\left(t_{0}, x\right)$ exists, where $t_{0}=(n+l-1) T^{+}$and $n T^{+}$, and is finite for $x \in R_{+}$and $n \in N$.

Lemma 6 (see [33]). Suppose $V \in V_{0}$ and

$$
D^{+} V(t, x) \leq g(t, V(t, x))
$$

$$
\begin{aligned}
& t \neq(n+l-1) T, t \neq n T, \\
& z^{*}(t)= \begin{cases}\frac{P \exp \left[-d_{1}\left(1+\varepsilon_{3}\right)(t-(n-1) T)\right]}{1-\left(1-\delta_{3}\right) \exp \left(-d_{1}\left(1+\varepsilon_{3}\right) T\right)}, & (n-1) T<t \leq(n+l-1) T, \\
\frac{P\left(1-\delta_{3}\right) \exp \left[-d_{1}\left(1+\varepsilon_{3}\right)(t-(n-1) T)\right]}{1-\left(1-\delta_{3}\right) \exp \left(-d_{1}\left(1+\varepsilon_{3}\right) T\right)}, & (n+l-1) T<t \leq n T,\end{cases}
\end{aligned}
$$

$n \in \aleph$, where $\aleph$ is set of natural numbers and

$$
\begin{aligned}
z^{*}\left(0^{+}\right) & =z^{*}\left(n T^{+}\right) \\
& =\frac{P}{1-\left(1-\delta_{3}\right) \exp \left(-d_{1}\left(1+\varepsilon_{3}\right) T\right)},
\end{aligned}
$$

$$
\begin{aligned}
z^{*}\left(l T^{+}\right) & =z^{*}\left((n+1-1) T^{+}\right) \\
& =\frac{P\left(1-\delta_{3}\right) \exp \left(-d_{1}\left(1+\varepsilon_{3}\right) l T\right)}{1-\left(1-\delta_{3}\right) \exp \left(-d_{1}\left(1+\varepsilon_{3}\right) T\right)}
\end{aligned}
$$

is a positive periodic solution of system (8). We obtain that
Using the lemma, we obtain the following result.

System (2) has a unique positive periodic solution $z^{*}(t)$ which is globally asymptotically stable, where where $g: R_{+} \times R_{+} \rightarrow R$ satisfies $(H)$ and $\vartheta_{n}^{1}, \vartheta_{n}^{2}: R_{+} \rightarrow R_{+}$are nondecreasing for all $n \in N$. Let $r(t)$ be the maximal solution for the impulsive Cauchy problem

$$
\begin{aligned}
\frac{d s}{d t} & =g(t, s(t)), \quad t \neq n T, \quad t \neq(n+l-1) T, \\
s\left(t^{+}\right) & =9_{n}^{1}(s(t)), \quad t=(n+l-1) T, \\
s\left(t^{+}\right) & =9_{n}^{2}(s(t)), \quad t=n T, \\
s\left(0^{+}\right) & =s_{0} \geq 0,
\end{aligned}
$$

Lemma 7. There exists a constant $M$ such that $x(t) \leq M$, $y(t) \leq M$, and $z(t) \leq M$ for each solution $(x(t), y(t), z(t))$

We use comparison theorem on impulsive differential equation:

$$
\begin{aligned}
\frac{d z(t)}{d t} & =-d_{1}\left(1+\varepsilon_{3}\right) z(t), \quad t \neq n T, \quad t \neq(n+l-1) T, \\
\Delta z & =-\delta_{3} z(t), \quad t=(n+l-1) T, \\
z\left(t^{+}\right) & =P, \quad t=n T, \\
z\left(0^{+}\right) & =z_{0} \geq 0 .
\end{aligned}
$$

defined on $[0, \infty)$. Then, $V\left(0^{+}, x_{0}\right) \leq s_{0}$ implies that $V(t, x(t)) \leq r(t), t \geq 0$, where $x(t)$ is any solution of (6).

$$
\begin{aligned}
& z(t) \\
& = \begin{cases}\left(1-\delta_{3}\right)^{n-1}\left(z\left(0^{+}\right)-\frac{P\left(1-\delta_{3}\right) e^{-T}}{1-\left(1-\delta_{3}\right) \exp \left(-d_{1}\left(1+\varepsilon_{3}\right) T\right)}\right) \exp \left(-d_{1}\left(1+\varepsilon_{3}\right) t\right)+z^{*}(t), & (n-1) T<t \leq(n+l-1) T, \\
\left(1-\delta_{3}\right)^{n}\left(z\left(0^{+}\right)-\frac{P\left(1-\delta_{3}\right) e^{-T}}{1-\left(1-\delta_{3}\right) \exp \left(-d_{1}\left(1+\varepsilon_{3}\right) T\right)}\right) \exp \left(-d_{1}\left(1+\varepsilon_{3}\right) t\right)+z^{*}(t), & (n+l-1) T<t \leq n T .\end{cases}
\end{aligned}
$$


Lemma 8. $\lim _{t \rightarrow \infty}\left|z^{*}(t)-z(t)\right|=0$ for all positive periodic solution $z(t)$ of system (2) with $z\left(0^{+}\right) \geq 0$.

Therefore, system (2) has a prey- (pest-) free periodic solution

$$
\left(0,0, z^{*}(t)\right)
$$

Next we find the expression of some critical parameters under the condition for all species persistence of system (1).

\section{Boundedness and Uniform Persistence}

Theorem 9. There exist position constants $M_{x}, M_{y}$, and $M_{z}$ for every position solution $(x(t), y(t), z(t))$ of system (1) with all sufficiently large $t$.

Proof. Assume that $(x(t), y(t), z(t))$ is an arbitrary positive solution of system (1); then from the first and second equations of system (1) we get

$$
\frac{d x(t)}{d t} \leq x(t)\left(a_{1}-x(t)\right)
$$

We have

$$
\lim \sup x(t) \leq a_{1}
$$

Similarly,

$$
\frac{d x(t)}{d t} \leq y(t)\left(a_{2}-y(t)\right)
$$

We have

$$
\lim \sup y(t) \leq a_{2}
$$

Let

$$
W=\frac{x(t)}{m_{2}}+\frac{y(t)}{m_{1}}+\frac{z(t)}{m_{1} m_{2}} .
$$

Then,

$$
\begin{aligned}
\frac{d W}{d t} & =\frac{1}{m_{2}} \frac{d x(t)}{d t}+\frac{1}{m_{2}} \frac{d y(t)}{d t}+\frac{1}{m_{1} m_{2}} \frac{d z(t)}{d t} \\
\frac{d W}{d t} & \leq \frac{x(t)}{m_{2}}\left(a_{1}-x(t)\right)+\frac{y(t)}{m_{1}}\left(a_{2}-y(t)\right)-\frac{d_{1} z(t)}{m_{1} m_{2}} \\
& \leq \frac{a_{1} x(t)}{m_{2}}+\frac{a_{2} y(t)}{m_{1}}-\frac{d_{1} z(t)}{m_{1} m_{2}}
\end{aligned}
$$

since $a_{1}>0, a_{2}>0$,

$$
\frac{d W}{d t} \leq \frac{2 a_{1}^{2}}{m_{2}}+\frac{2 a_{2}^{2}}{m_{1}}-\delta W
$$

where

$$
\begin{aligned}
\delta & =\min \left\{\frac{1}{m_{2}}, \frac{1}{m_{1}}, \frac{d_{1}}{m_{1} m_{2}}\right\}, \\
\frac{d W}{d t}+\delta W & \leq 2\left(\frac{a_{1}^{2}}{m_{2}}+\frac{a_{2}^{2}}{m_{1}}\right) .
\end{aligned}
$$

According to the theorem on differential inequalities [35], we have

$$
0 \leq W(x, y, z) \leq \frac{2}{\delta}+\frac{W(x(0), y(0), z(0))}{e^{\delta t}}
$$

and, for $t \rightarrow \infty$,

$$
0 \leq W \leq \frac{2}{\delta}
$$

Thus, all solutions of system (1) enter into the region

$$
\begin{aligned}
\widehat{B} & =\left\{(x(t), y(t), z(t)): 0 \leq W \leq \frac{2}{\delta}+\epsilon, \text { for any } \epsilon\right. \\
& >0\} .
\end{aligned}
$$

This proves the theorem.

Note 2. Since $W$ is bounded above, all of $x(t), y(t), z(t)$ are bounded above. Therefore there exist positive constants $M_{x}, M_{y}, M_{z}$, and $T_{1}$ such that $x(t) \leq M_{x}, y(t) \leq M_{y}$, and $z(t) \leq M_{z}$ for $t \geq T_{1}$.

Theorem 10. If $W_{1}=\left[a_{1}\left(1-\widehat{\epsilon}_{1}\right)-M_{x}\right]>0, W_{2}=\left\{a_{2}\left(1-\widehat{\epsilon}_{2}\right)-\right.$ $\left.M_{y}\right\}>0$, and $W_{3}=\left(m_{1} m_{x}^{p} /\left(1+e_{1} M_{x}^{q}\right)+m_{1} m_{y}^{s} /\left(1+e_{2} M_{y}^{r}\right)-\right.$ $\left.d_{1}\left(1+\widehat{\epsilon}_{3}\right)\right)>0$ for every small constant $\epsilon_{i}(i=1,2,3)>0$, then system (1) is uniformly persistent.

Proof. From Theorem 9, there exist positive constants $M_{x}, M_{y}, M_{z}$, and $T_{3}=\max \left\{T_{1}, T_{2}\right\}>0$ such that $x(t) \leq$ $M_{x}, y(t) \leq M_{y}, z(t) \leq M_{z}$ for $t \geq T_{3}$. From the first equation of system (1), we get

$$
\begin{aligned}
\frac{d x(t)}{d t} \geq & x(t)\left\{a_{1}-M_{x}\right\}-\left\{\frac{M_{z}}{2 \sqrt{e_{1}}}\right\}\{x(t)\}^{p-q / 2} \\
\geq & x(t)\left\{a_{1}\left(1-\widehat{\epsilon}_{1}\right)-M_{x}\right\} \\
& -\left\{\frac{M_{z}}{2 \sqrt{e_{1}}}\right\}\{x(t)\}^{p-q / 2} .
\end{aligned}
$$

From Lemma 2, if $W_{1}=\left[a_{1}\left(1-\widehat{\epsilon}_{1}\right)-M_{x}\right]>0$, then there is positive constant $T_{4}$ such that

$$
\begin{aligned}
& x(t) \geq\left\{\frac{2 \sqrt{e_{1}}\left[a_{1}\left(1-\widehat{\epsilon}_{1}\right)-M_{x}\right]}{M_{z}}\right\}^{1 /(p-q / 2)} \\
& \cdot {\left[1+\frac{2 \sqrt{e_{1}}\left[a_{1}\left(1-\widehat{\epsilon}_{1}\right)-M_{x}\right]\left\{x\left(T_{4}\right)\right\}^{-(p-q / 2)}}{M_{z}}\right] } \\
&:=m_{x}
\end{aligned}
$$

for $t \geq T_{4}$. From the second equation of (1) we obtain that $\left.d y(t) / d t \geq y(t)\left\{a_{2}\left(1-\widehat{\epsilon}_{2}\right)-M_{y}\right\}-M_{z}\{y(t)\}^{p-q / 2} / 2 \sqrt{e_{2}}\right\}$. It 
is known that if $W_{2}=\left\{a_{2}\left(1-\widehat{\epsilon}_{2}\right)-M_{y}\right\}>0$, there are two positive constants $T_{5}$ and $m_{y}$ such that

$$
\begin{aligned}
& y(t)\left.\geq\left\{\frac{2 \sqrt{e_{2}}\left[a_{2}\left(1-\widehat{\epsilon}_{2}\right)-M_{y}\right]}{M_{z}}\right\}\right\}^{1 /(s-r / 2)} \\
& \cdot {\left[1+\frac{2 \sqrt{e_{2}}\left[a_{2}\left(1-\widehat{\epsilon}_{2}\right)-M_{y}\right]\left\{y\left(T_{5}\right)\right\}^{-(s-r / 2)}}{M_{z}}\right] } \\
&:=m_{y}
\end{aligned}
$$

for $t \geq T_{5}$. From the third equation of system (1), we get $d z(t) / d t \geq\left\{m_{1} m_{x}^{p} /\left(1+e_{1} M_{x}^{q}\right)+m_{1} m_{y}^{s} /\left(1+e_{2} M_{y}^{r}\right)-d_{1}(1+\right.$ $\left.\left.\widehat{\epsilon}_{3}\right)\right\} z(t)$. We know that if $W_{3}=\left(m_{1} m_{x}^{p} /\left(1+e_{1} M_{x}^{q}\right)+m_{1} m_{y}^{s} /(1+\right.$ $\left.\left.e_{2} M_{y}^{r}\right)-d_{1}\left(1+\widehat{\epsilon}_{3}\right)\right)>0$, there are two positive constants $T_{6}$ and $m_{z}$ such that $z(t) \geq m_{z}$ for $t \geq T_{6}$. Let $T=\max \left\{T_{3}, T_{4}, T_{5}, T_{6}\right\}$; then we have $m_{x} \leq x(t) \leq M_{x}, m_{y} \leq y(t) \leq M_{y}, m_{z} \leq$ $z(t) \leq M_{z}$ for $t \geq T$. This completes the proof.

\section{Stability of a Periodic Solution with Prey Eradication}

In this section, we examine the stability of the pest eradication periodic solution $\left(0,0, z^{*}(t)\right)$ of system (2). We find stability analysis of system (2) for different values of $p, q$, $s$, and $r$. We have system (2) that represents two-prey and one-predator model, (i) with Holling type II functional responses for $p=$ $q=s=r=1$, (ii) with Holling type III functional responses for $p=q=s=r=2$, (iii) with Holling type IV functional response for $p=1, q=2, s=1, r=2$, (iv) with mixed Holling type II-III functional responses for $p=q=1$ and $s=r=2$, (v) with mixed Holling type II-IV functional responses for $p=q=s=1$ and $r=2$, and (vi) with mixed Holling type III-IV functional responses for $p=q=r=2$ and $s=1$. We find that Holling type II, Holling type IV, and mixed Holling types II-IV system have same Jacobian matrix for the solutions $\left(0,0, z^{*}(t)\right)$ and Holling type III, mixed Holling types II-III, and Holling type III-IV systems have different Jacobian matrices for the solutions $\left(0,0, z^{*}(t)\right)$.

Theorem 11. Let $(x(t), y(t), z(t))$ be any solution of system (3) with Holling type II, Holling type IV, and mixed Holing II-IV functional responses; then $\left(0,0, z^{*}(t)\right)$ is locally asymptotically stable if

$$
\begin{aligned}
& \frac{a_{i}\left(1+\varepsilon_{i}\right) T}{c_{i}}-\frac{P\left[(1-A)+\left(1-\delta_{3}\right)(A-B)\right]}{d_{1}\left(1+\varepsilon_{3}\right)\left(1-\left(1-\delta_{3}\right) B\right)} \\
& \quad<\frac{1}{c_{i}} \ln \left(\frac{1}{\left(1-\delta_{i}\right)}\right)
\end{aligned}
$$

for $i=1,2$ where $A=\exp \left(-d_{1}\left(1+\varepsilon_{3}\right) l T\right), B=\exp \left(-d_{1}(1+\right.$ $\left.\left.\varepsilon_{3}\right) T\right)$.

Proof. The local stability of the periodic solution $\left(0,0, z^{*}(t)\right)$ may be determined by considering the behavior of small amplitude perturbations of the solution. Define $x(t)=u(t)$, $y(t)=v(t)$, and $z(t)=w(t)+z^{*}(t)$.

From system (3), we have the following linear system:

$$
\left(\begin{array}{c}
u(t) \\
v(t) \\
w(t)
\end{array}\right)=\Phi(t)\left(\begin{array}{c}
u(0) \\
v(0) \\
w(0)
\end{array}\right),
$$

where $\Phi(t)$ satisfies

$$
\frac{d \phi}{d t}=\left(\begin{array}{ccc}
\left(a_{1}\left(1+\varepsilon_{1}\right)-c_{1} z^{*}(t)\right) & 0 & 0 \\
0 & \left(a_{2}\left(1+\varepsilon_{2}\right)-c_{2} z^{*}(t)\right) & 0 \\
m_{1} z^{*}(t) & m_{2} z^{*}(t) & -d_{1}\left(1+\varepsilon_{3}\right)
\end{array}\right) \Phi(t)
$$

and $\Phi(0)=I$, where $I$ is the identity matrix. So the fundamental solution matrix is

$$
\Phi(t)=\left(\begin{array}{ccc}
\exp \left(\int_{0}^{t}\left(a_{1}\left(1+\varepsilon_{1}\right)-c_{1} z^{*}(s)\right) d s\right) & 0 & 0 \\
0 & \exp \left(\int_{0}^{t}\left(a_{2}\left(1+\varepsilon_{2}\right)-c_{2} z^{*}(s)\right) d s\right) & 0 \\
\exp \left(\int_{0}^{t} m_{1} z^{*}(s) d s\right) & \exp \left(\int_{0}^{t} m_{2} z^{*}(s) d s\right) & \exp \left(\int_{0}^{t}-d_{1}\left(1+\varepsilon_{3}\right) d s\right)
\end{array}\right) .
$$




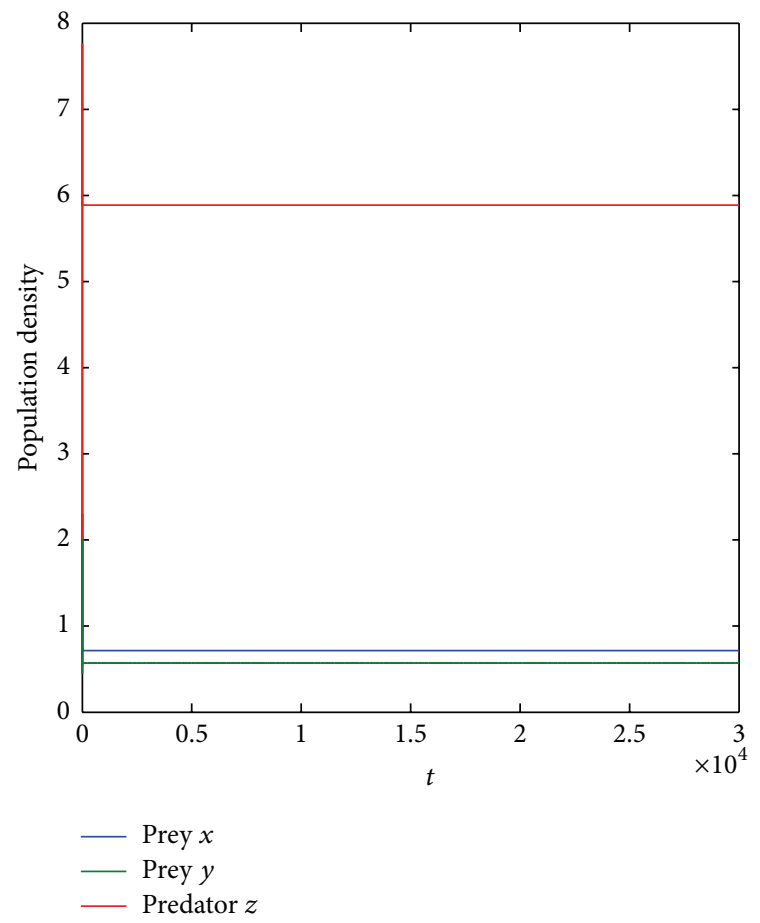

(a)

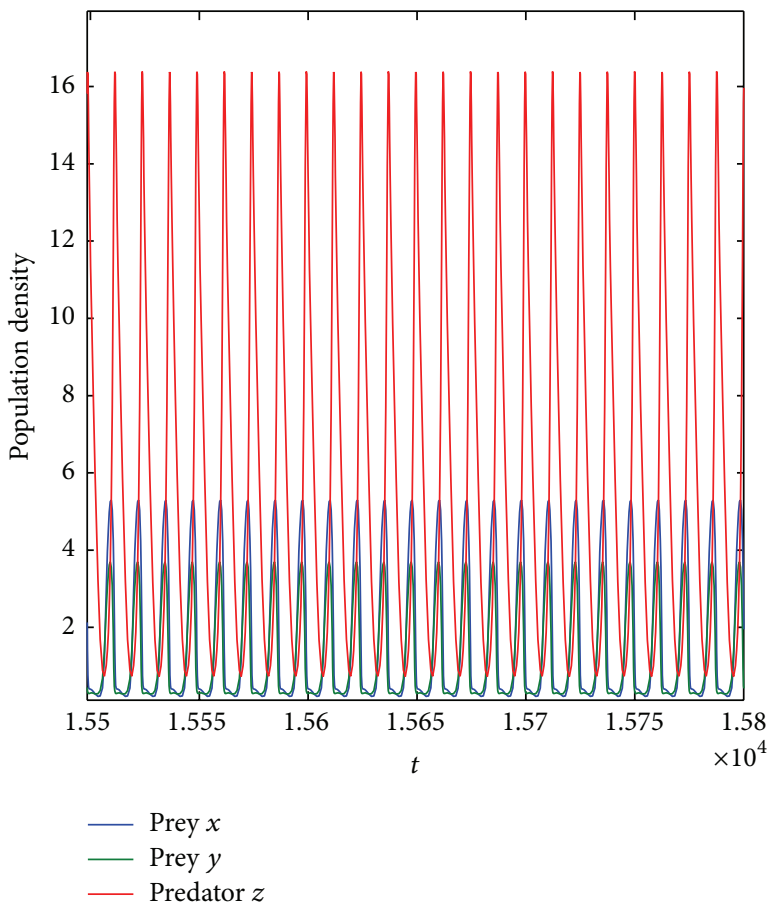

(b)

FIGURE 1: Solution trajectories of system (1) with Holling type III responses ( $p=2, q=2, s=2$, and $r=2)$ showing (a) stable behavior in absence of seasonal perturbations and (b) periodic behavior in presence of seasonal perturbation with data set (45).
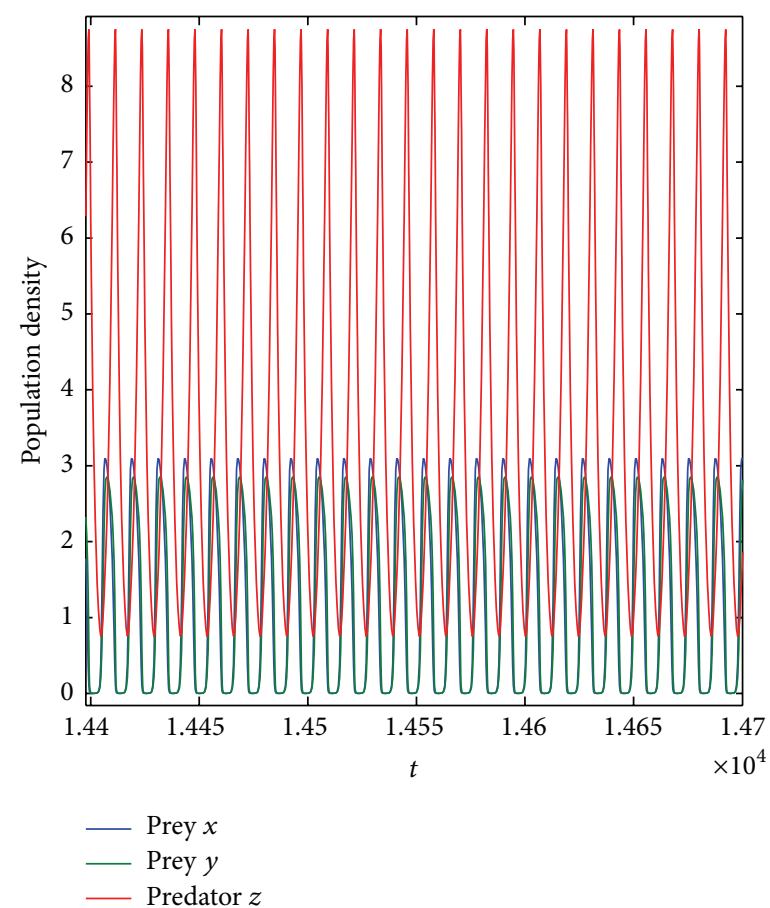

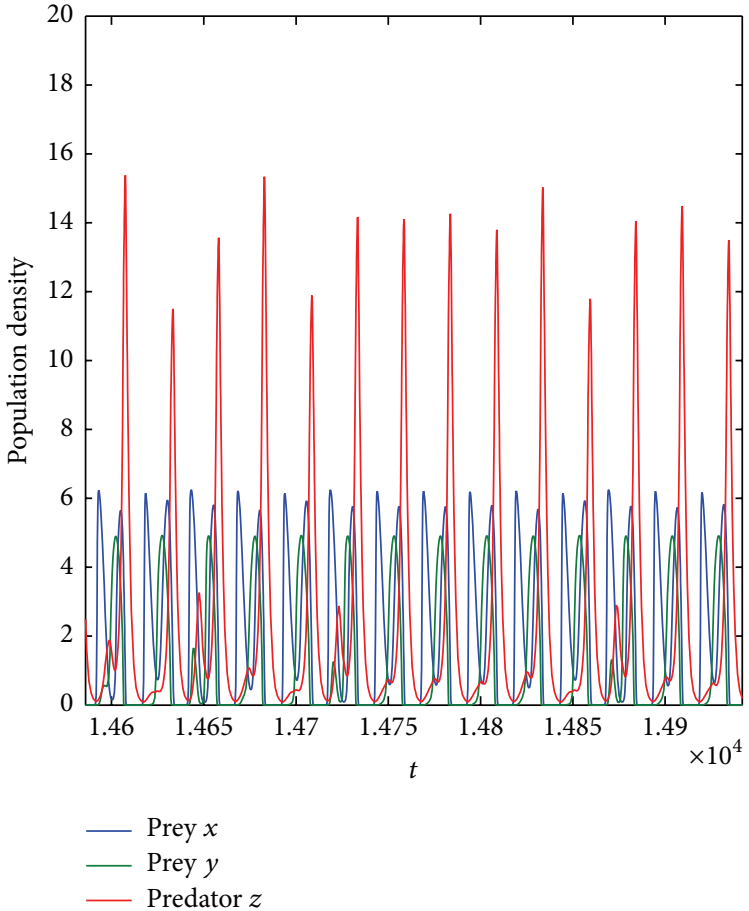

(b)

FIGURE 2: Solution trajectories of system (1) with mixed Holling type II-IV responses ( $p=1, q=1, s=1$, and $r=2)$ showing (a) periodic behavior in absence of seasonal perturbation and (b) quasiperiodic behavior in presence of seasonal perturbation with data set (45). 


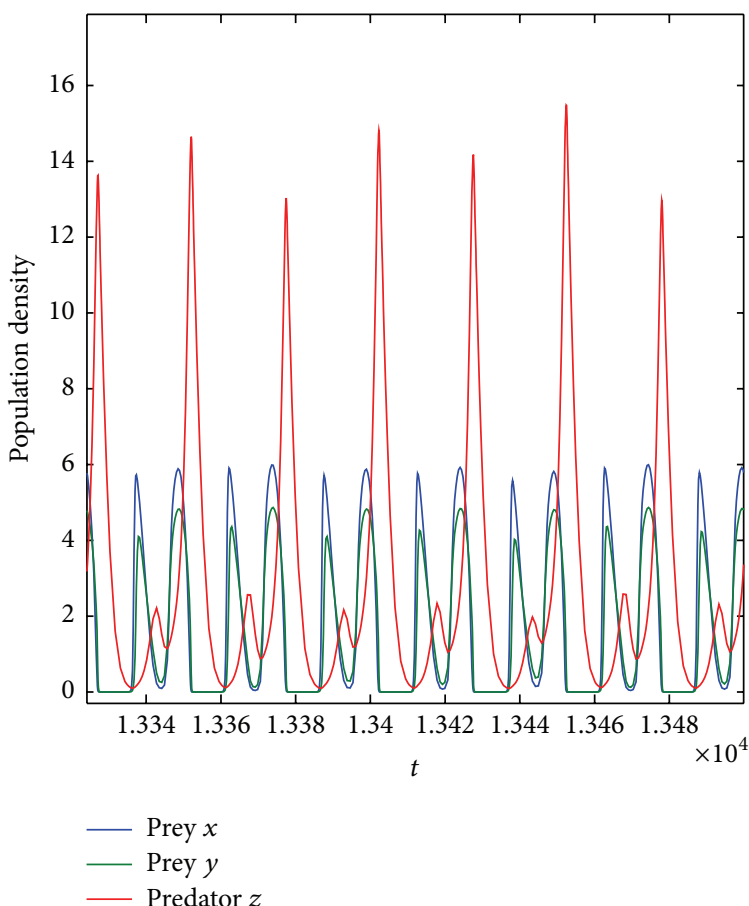

(a)

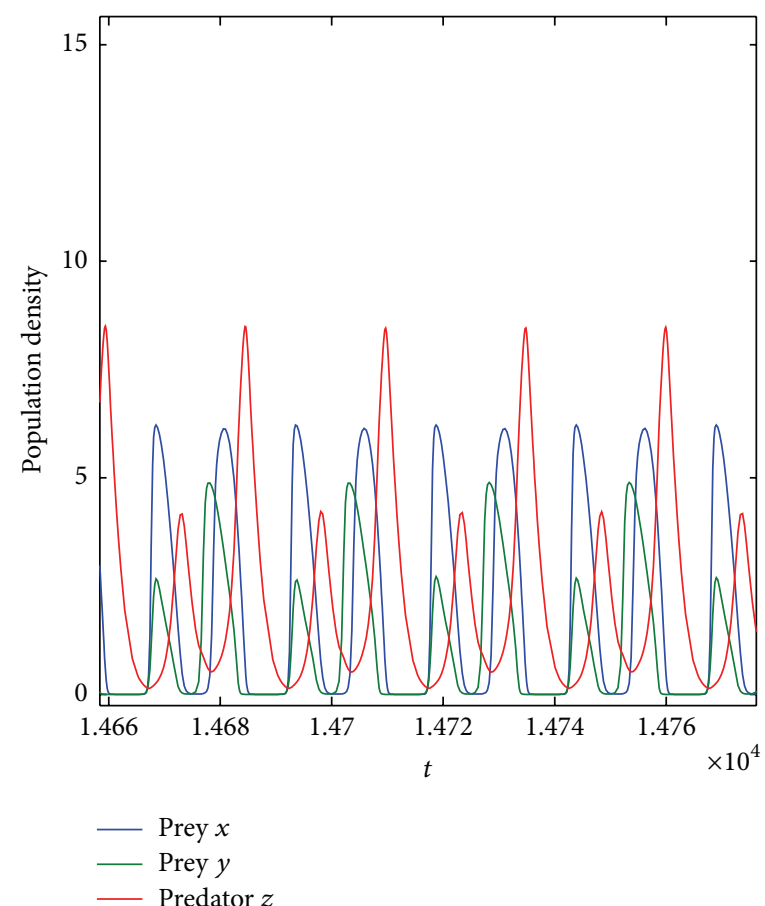

(b)

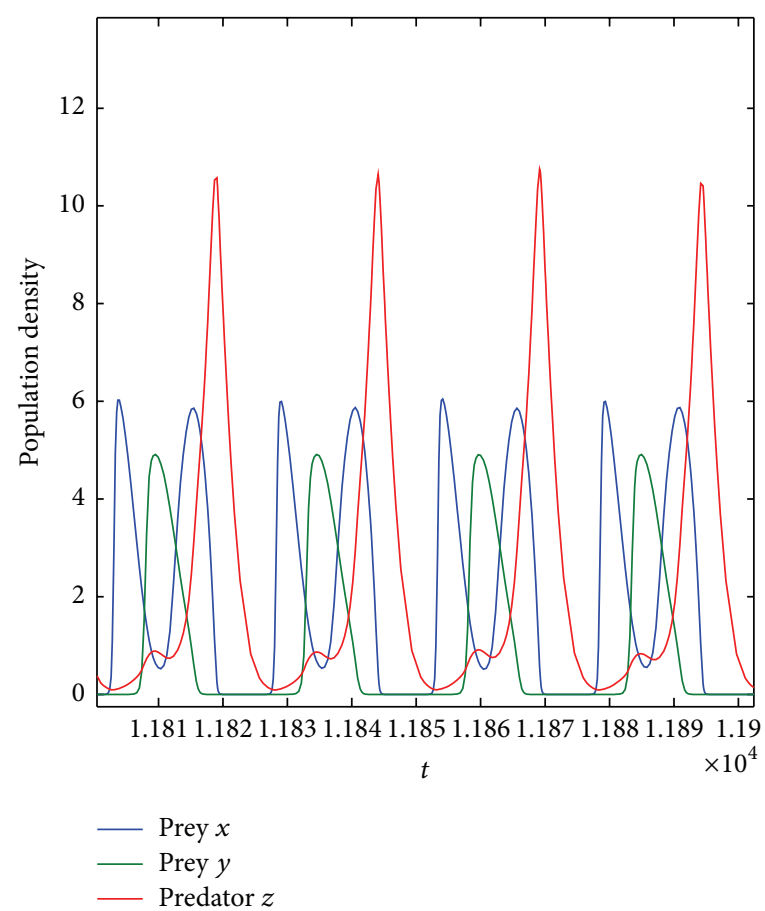

(c)

FIgURE 3: Solution trajectories of system (1) with mixed Holling type II-IV responses ( $p=1, q=1, s=1$, and $r=2)$ showing (a) in-phase synchronous variation for $\psi=0$, (b) out-phase synchronous variation for $\psi=\pi / 2$, and (c) antiphase synchronous variation for $\psi=\pi$ with $\phi=\pi / 2$ and data set (45). 


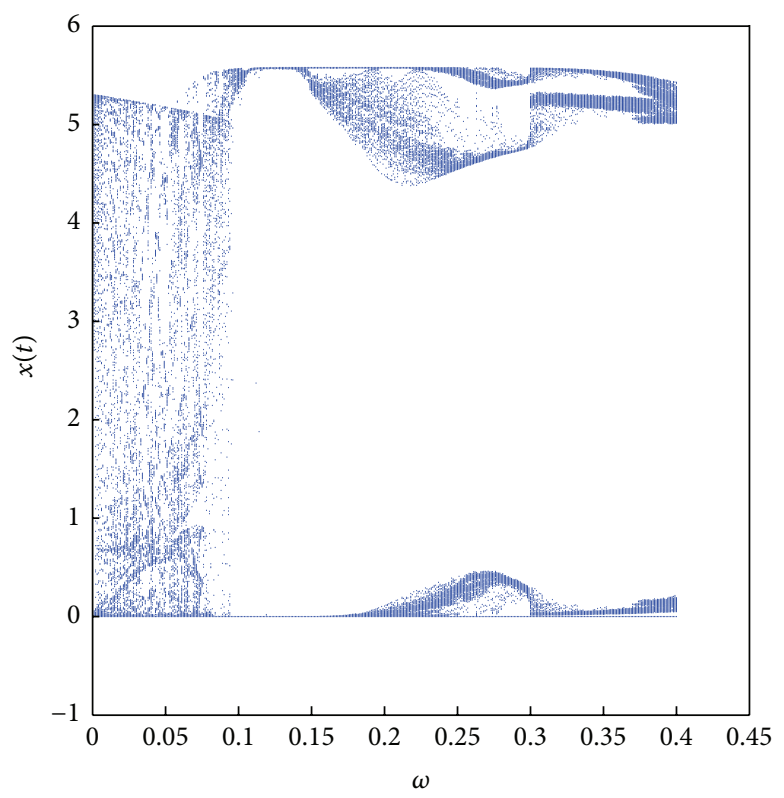

(a)

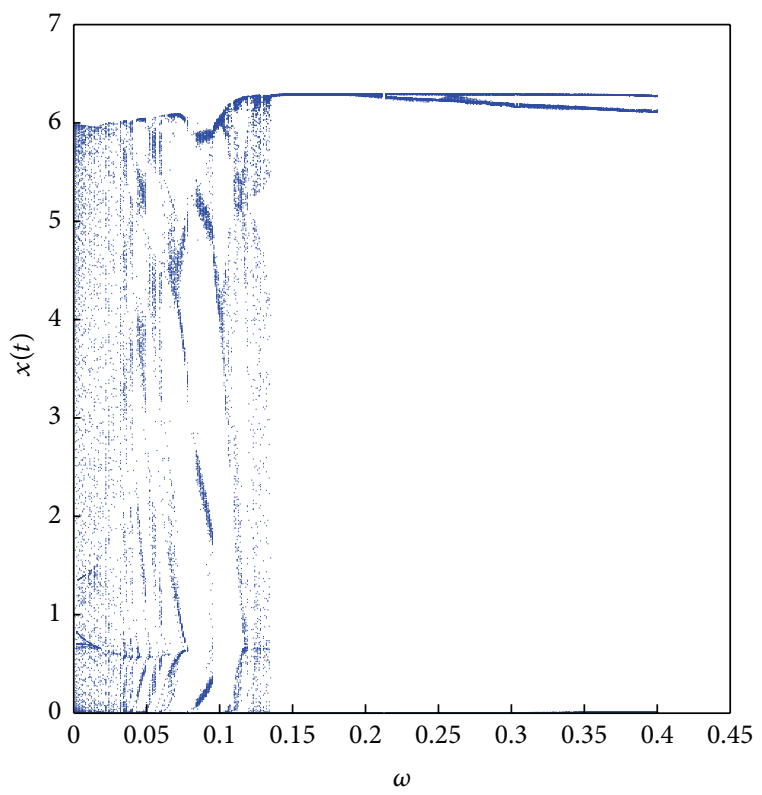

(b)

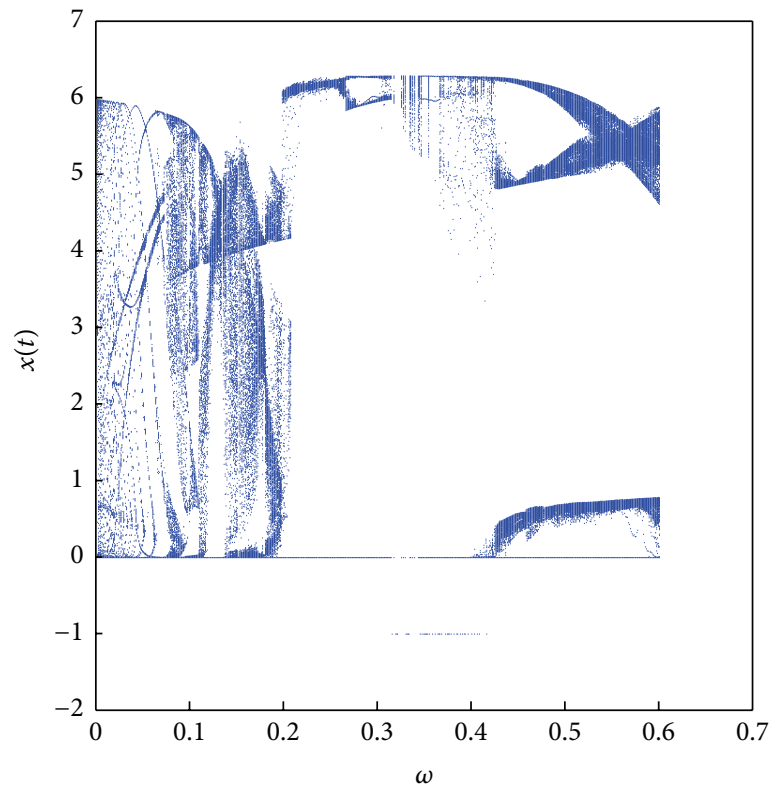

(c)

FIGURE 4: Bifurcation diagram of system (1) with mixed Holling type II-IV functional responses with respect to seasonal parameter $\omega$ for (a) $\psi=0, \phi=\pi / 2$, (b) $\psi=\pi / 2, \phi=\pi / 2$, and (c) $\psi=\pi, \phi=\pi / 2$ with data set (45).

The resetting impulsive conditions of system (28) become

$$
\begin{aligned}
& \left(\begin{array}{c}
u\left((n+l-1) T^{+}\right) \\
v\left((n+l-1) T^{+}\right) \\
w\left((n+l-1) T^{+}\right)
\end{array}\right) \\
& =\left(\begin{array}{ccc}
\left(1-\delta_{1}\right) & 0 & 0 \\
0 & \left(1-\delta_{2}\right) & 0 \\
0 & 0 & \left(1-\delta_{3}\right)
\end{array}\right)\left(\begin{array}{c}
u((n+l-1) T) \\
v((n+l-1) T) \\
w((n+l-1) T)
\end{array}\right), \\
& \left(\begin{array}{c}
u\left(n T^{+}\right) \\
v\left(n T^{+}\right) \\
w\left(n T^{+}\right)
\end{array}\right)=\left(\begin{array}{lll}
1 & 0 & 0 \\
0 & 1 & 0 \\
0 & 0 & 1
\end{array}\right)\left(\begin{array}{c}
u(n T) \\
v(n T) \\
w(n T)
\end{array}\right) .
\end{aligned}
$$

Note that all eigenvalues of the matrix

$S$

$$
=\left(\begin{array}{ccc}
\left(1-\delta_{1}\right) & 0 & 0 \\
0 & \left(1-\delta_{2}\right) & 0 \\
0 & 0 & \left(1-\delta_{3}\right)
\end{array}\right)\left(\begin{array}{lll}
1 & 0 & 0 \\
0 & 1 & 0 \\
0 & 0 & 1
\end{array}\right) \Phi(T)
$$

are $\mu_{1}=\left(1-\delta_{1}\right) \exp \left(\int_{0}^{T}\left(a_{1}\left(1+\varepsilon_{1}\right)-c_{1} z^{*}(t)\right) d t\right), \mu_{2}=(1-$ $\left.\delta_{2}\right) \exp \left(\int_{0}^{T}\left(a_{2}\left(1+\varepsilon_{2}\right)-c_{2} z^{*}(t)\right) d t\right)$, and $\mu_{3}=\exp \left(\int_{0}^{T}-d_{1}(1+\right.$ $\left.\left.\varepsilon_{3}\right) d t\right)=\exp \left(-d_{1}\left(1+\varepsilon_{3}\right) T\right)<1$. 


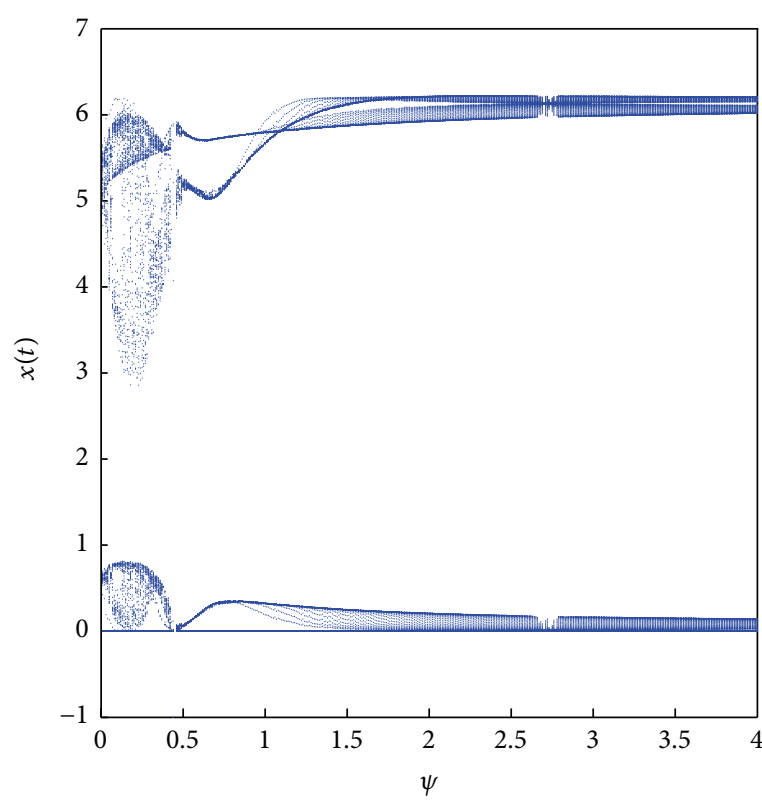

(a)

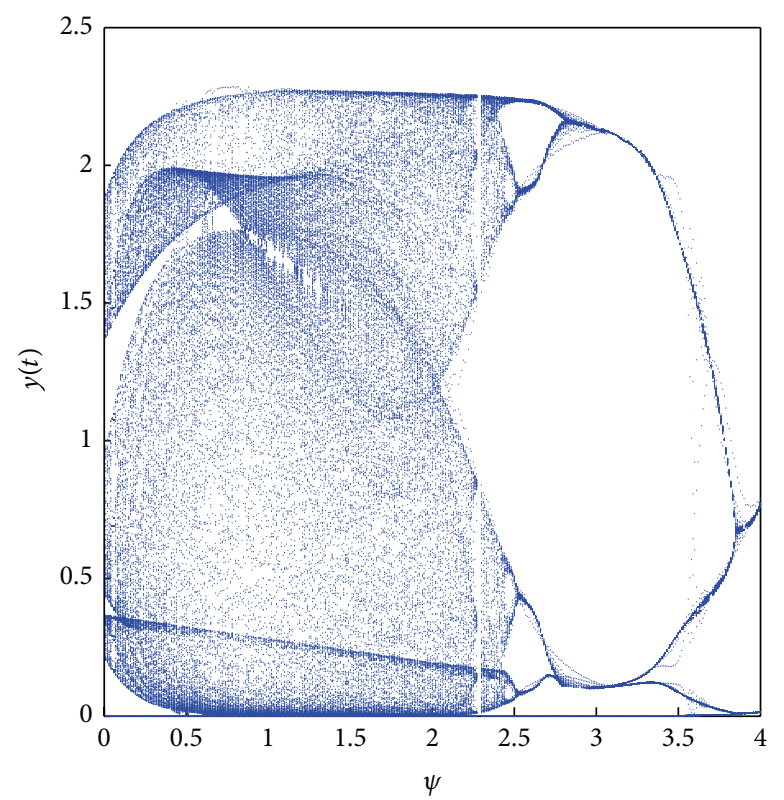

(b)

FIGURE 5: Bifurcation diagram of system (1) (a) with mixed Holling type II-IV responses and (b) with Holling type IV responses with respect to seasonal parameter $\psi$ with data set $(45)$.

So $\left|\mu_{1}\right|<1,\left|\mu_{2}\right|<1$, if and only if

$$
\begin{aligned}
& \frac{a_{1}\left(1+\varepsilon_{1}\right) T}{c_{1}}-\frac{P\left[(1-A)+\left(1-\delta_{3}\right)(A-B)\right]}{d_{1}\left(1+\varepsilon_{3}\right)\left(1-\left(1-\delta_{3}\right) B\right)} \\
& <\frac{1}{c_{1}} \ln \left(\frac{1}{\left(1-\delta_{1}\right)}\right), \\
& \frac{a_{2}\left(1+\varepsilon_{2}\right) T}{c_{2}}-\frac{P\left[(1-A)+\left(1-\delta_{3}\right)(A-B)\right]}{d_{1}\left(1+\varepsilon_{3}\right)\left(1-\left(1-\delta_{3}\right) B\right)} \\
& <\frac{1}{c_{2}} \ln \left(\frac{1}{\left(1-\delta_{2}\right)}\right),
\end{aligned}
$$

where $A=\exp \left(-d_{1}\left(1+\varepsilon_{3}\right) l T\right), B=\exp \left(-d_{1}\left(1+\varepsilon_{3}\right) T\right)$.

According to Floquet theory (see [34]) of impulsive differential equation, the two-pest eradication solution $\left(0,0, z^{*}(t)\right)$ is locally asymptotically stable. This completes the proof.

Theorem 12. Let $(x(t), y(t), z(t))$ be any solution of (3) with Holling type III; then periodic solution $\left(0,0, z^{*}(t)\right)$ is locally asymptotically stable if

$$
T<\frac{1}{a_{i}\left(1+\varepsilon_{i}\right)} \ln \left(\frac{1}{\left(1-\delta_{i}\right)}\right) \quad \text { for } i=1,2 .
$$

Proof. Let us take the transformation $x_{1}(t)=u_{1}(t), x_{2}(t)=$ $v_{1}(t), y(t)=w_{1}(t)+z^{*}(t)$; then the system becomes

$$
\left(\begin{array}{l}
u_{1}(t) \\
v_{1}(t) \\
w_{1}(t)
\end{array}\right)=\Phi(t)\left(\begin{array}{l}
u_{1}(0) \\
v_{1}(0) \\
w_{1}(0)
\end{array}\right),
$$

where $\Phi(t)$ satisfies

$$
\begin{aligned}
& \frac{d \phi}{d t} \\
& =\left(\begin{array}{ccc}
a_{1}\left(1+\varepsilon_{1}\right) & 0 & 0 \\
0 & a_{2}\left(1+\varepsilon_{2}\right) & 0 \\
0 & 0 & -d_{1}\left(1+\varepsilon_{3}\right)
\end{array}\right) \Phi(t) .
\end{aligned}
$$

Using Floquet theory (see [34]), we find that the following inequalities hold:

$$
\begin{aligned}
& T<\frac{1}{a_{1}\left(1+\varepsilon_{1}\right)} \ln \left(\frac{1}{\left(1-\delta_{1}\right)}\right), \\
& T<\frac{1}{a_{2}\left(1+\varepsilon_{2}\right)} \ln \left(\frac{1}{\left(1-\delta_{2}\right)}\right) .
\end{aligned}
$$

Therefore, $\left(0,0, z^{*}(t)\right)$ is locally asymptotically stable. The proof is completed.

Theorem 13. The periodic solution $\left(0,0, z^{*}(t)\right)$ of system (3) with mixed Holling type II-III functional responses is locally asymptotically stable if

$$
\begin{aligned}
& \frac{a_{1}\left(1+\varepsilon_{1}\right) T}{c_{1}}-\frac{P\left[(1-A)+\left(1-\delta_{3}\right)(A-B)\right]}{d_{1}\left(1+\varepsilon_{3}\right)\left(1-\left(1-\delta_{3}\right) B\right)} \\
& \quad<\frac{1}{c_{1}} \ln \left(\frac{1}{\left(1-\delta_{1}\right)}\right), \\
& T<\frac{1}{a_{2}\left(1+\varepsilon_{2}\right)} \ln \left(\frac{1}{\left(1-\delta_{2}\right)}\right),
\end{aligned}
$$

where $A=\exp \left(-d_{1}\left(1+\varepsilon_{3}\right) l T\right), B=\exp \left(-d_{1}\left(1+\varepsilon_{3}\right) T\right)$. 


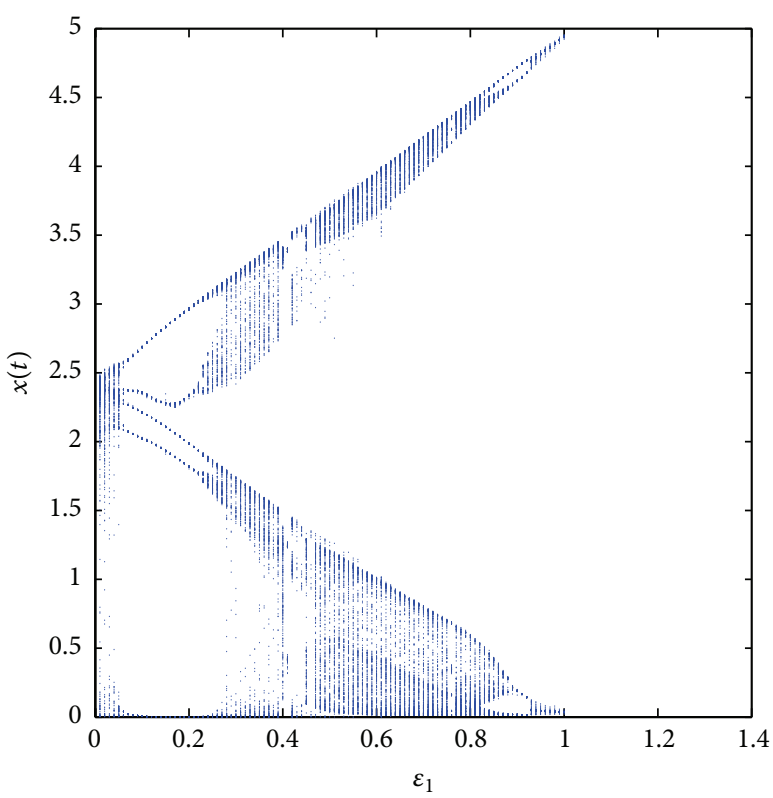

(a)

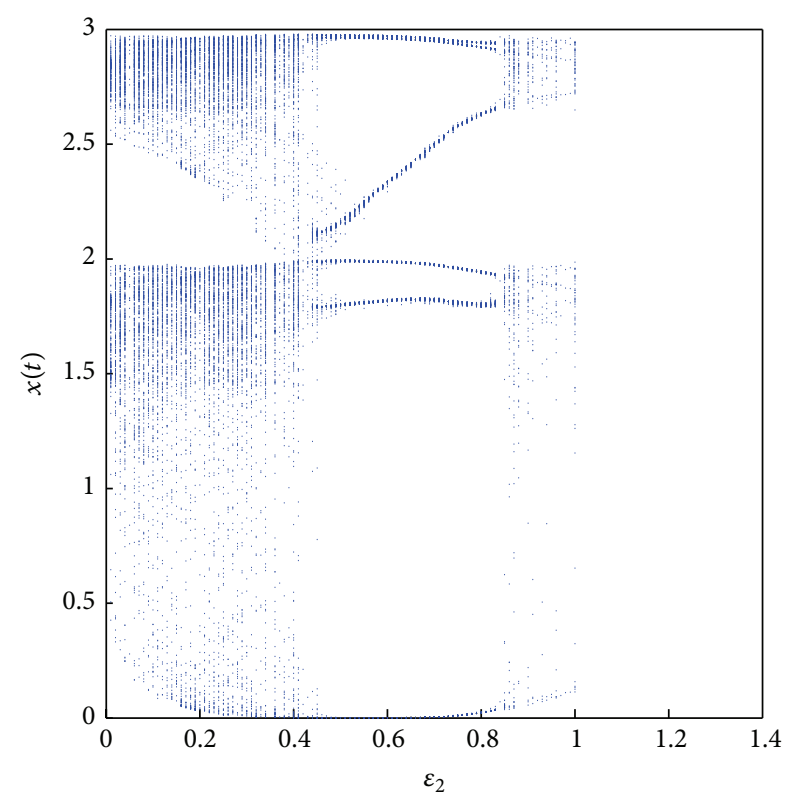

(b)

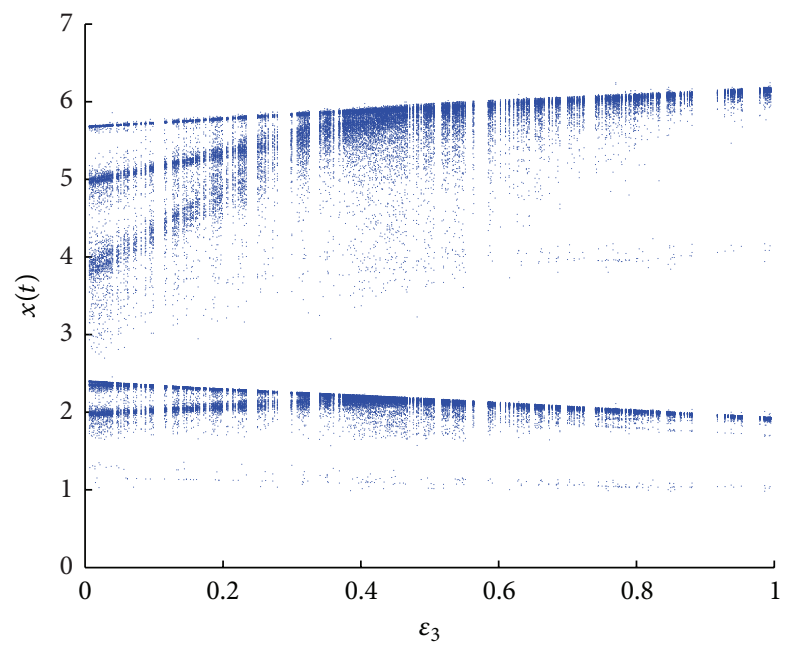

(c)

FIGURE 6: (a) Bifurcation diagram of system (1) with Holling type IV functional responses with respect to seasonal parameters (a) $\varepsilon_{1}$, (b) $\varepsilon_{2}$, and (c) $\varepsilon_{3}$ with data set (45).

Proof. Let us consider the transformation $x_{1}(t)=$ $u_{1}(t), x_{2}(t)=v_{1}(t), y(t)=w_{1}(t)+z^{*}(t)$; then the liberalization of the system is

$$
\left(\begin{array}{l}
u_{1}(t) \\
v_{1}(t) \\
w_{1}(t)
\end{array}\right)=\Phi(t)\left(\begin{array}{c}
u_{1}(0) \\
v_{1}(0) \\
w_{1}(0)
\end{array}\right),
$$

where $\Phi(t)$ satisfies

$$
\begin{aligned}
& \frac{d \phi}{d t} \\
& =\left(\begin{array}{ccc}
a_{1}\left(1+\varepsilon_{1}\right)-c_{1} z^{*}(t) & 0 & 0 \\
0 & a_{2}\left(1+\varepsilon_{2}\right) & 0 \\
m_{1} z^{*}(t) & m_{2} z^{*}(t) & -d_{1}\left(1+\varepsilon_{3}\right)
\end{array}\right) \Phi(t) .
\end{aligned}
$$

Applying Floquet theory (see [34]), the eigenvalues

$$
\begin{aligned}
& H \\
& =\left(\begin{array}{ccc}
\left(1-\delta_{1}\right) & 0 & 0 \\
0 & \left(1-\delta_{2}\right) & 0 \\
0 & 0 & \left(1-\delta_{3}\right)
\end{array}\right)\left(\begin{array}{lll}
1 & 0 & 0 \\
0 & 1 & 0 \\
0 & 0 & 1
\end{array}\right) \phi(T)
\end{aligned}
$$
are $\mu_{1}=\left(1-\delta_{1}\right) \exp \left(\int_{0}^{T}\left(a_{1}\left(1+\varepsilon_{1}\right)-c_{1} z^{*}(t)\right) d t\right), \mu_{2}=(1-$ $\left.\delta_{2}\right) \exp \left(\int_{0}^{T} a_{2}\left(1+\varepsilon_{2}\right) d t\right)=\left(1-\delta_{2}\right) \exp \left(a_{2}\left(1+\varepsilon_{2}\right) T\right)$, and $\mu_{3}=$ $\exp \left(\int_{0}^{T}-d_{1}\left(1+\varepsilon_{3}\right) d t\right)=\exp \left(-d_{1}\left(1+\varepsilon_{3}\right) T\right)<1$. 


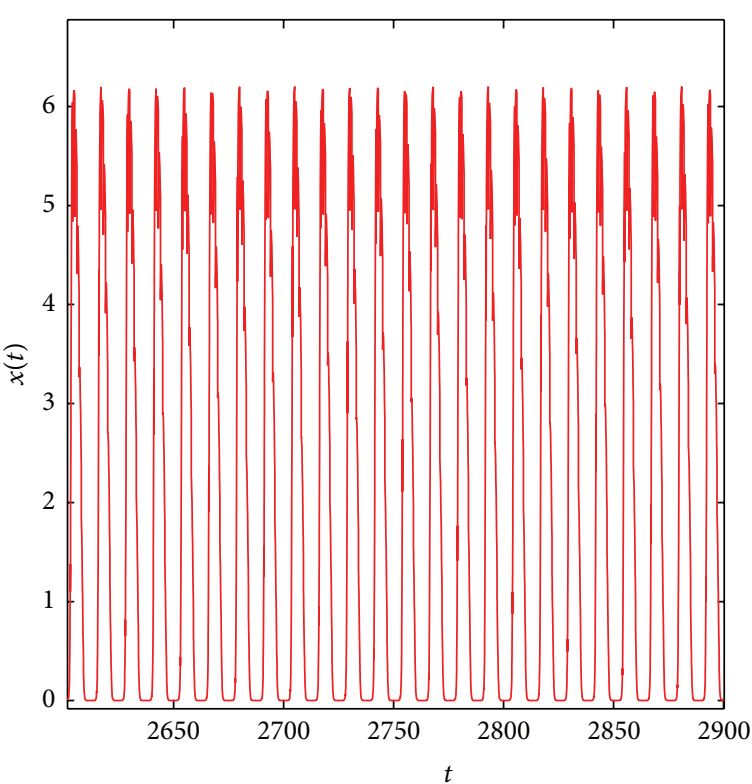

(a)

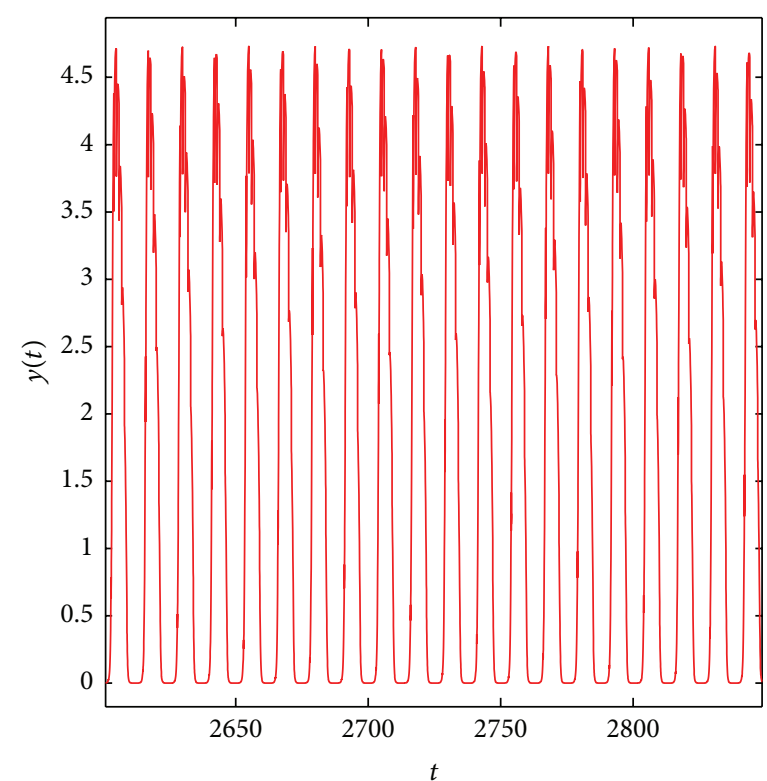

(b)

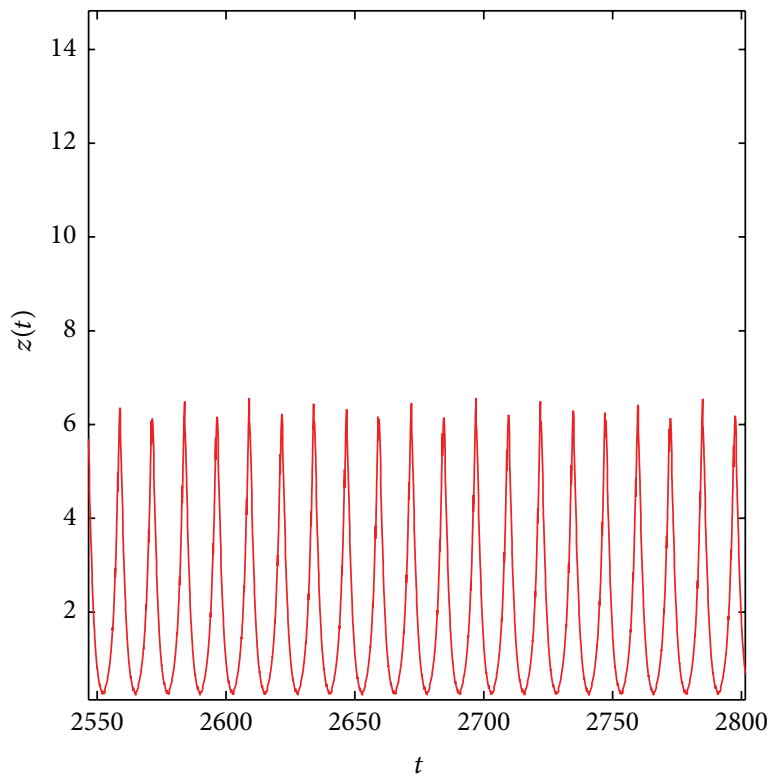

(c)

Figure 7: (a)-(c) Time series of prey $x(t)$ and $y(t)$ and predator $z(t)$ depicting coexisting populations of system (2) with mixed Holling type II-IV functional responses in presence of seasonal perturbation with data set (45) and $P=0.1$.

By using Floquet theory, $\left|\mu_{1}\right|<1,\left|\mu_{2}\right|<1$ if and only if

$$
\begin{aligned}
& \frac{a_{1}\left(1+\varepsilon_{1}\right) T}{c_{1}}-\frac{P\left[(1-A)+\left(1-\delta_{3}\right)(A-B)\right]}{d_{1}\left(1+\varepsilon_{3}\right)\left(1-\left(1-\delta_{3}\right) B\right)} \\
& <\frac{1}{c_{1}} \ln \left(\frac{1}{\left(1-\delta_{1}\right)}\right),
\end{aligned}
$$$$
T<\frac{1}{a_{2}\left(1+\varepsilon_{2}\right)} \ln \left(\frac{1}{\left(1-\delta_{2}\right)}\right) \text {, }
$$

where $A=\exp \left(-d_{1}\left(1+\varepsilon_{3}\right) l T\right), B=\exp \left(-d_{1}\left(1+\varepsilon_{3}\right) T\right)$. Therefore $\left(0,0, z^{*}(t)\right)$ is locally asymptotically stable if

$$
\begin{aligned}
& \frac{a_{1}\left(1+\varepsilon_{1}\right) T}{c_{1}}-\frac{P\left[(1-A)+\left(1-\delta_{3}\right)(A-B)\right]}{d_{1}\left(1+\varepsilon_{3}\right)\left(1-\left(1-\delta_{3}\right) B\right)} \\
& \quad<\frac{1}{c_{1}} \ln \left(\frac{1}{\left(1-\delta_{1}\right)}\right), \\
& T<\frac{1}{a_{2}\left(1+\varepsilon_{2}\right)} \ln \left(\frac{1}{\left(1-\delta_{2}\right)}\right) .
\end{aligned}
$$

The proof is completed. 


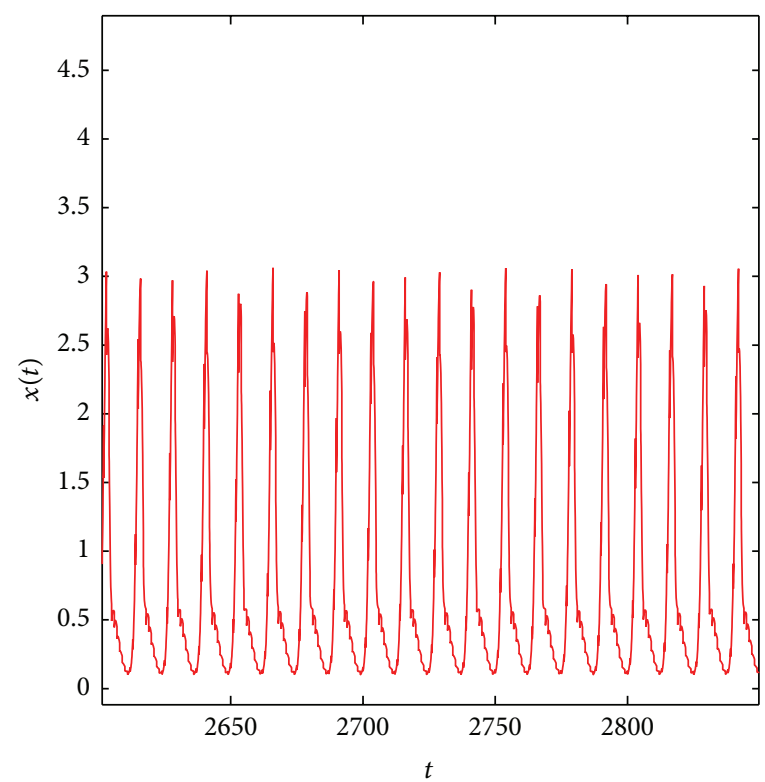

(a)

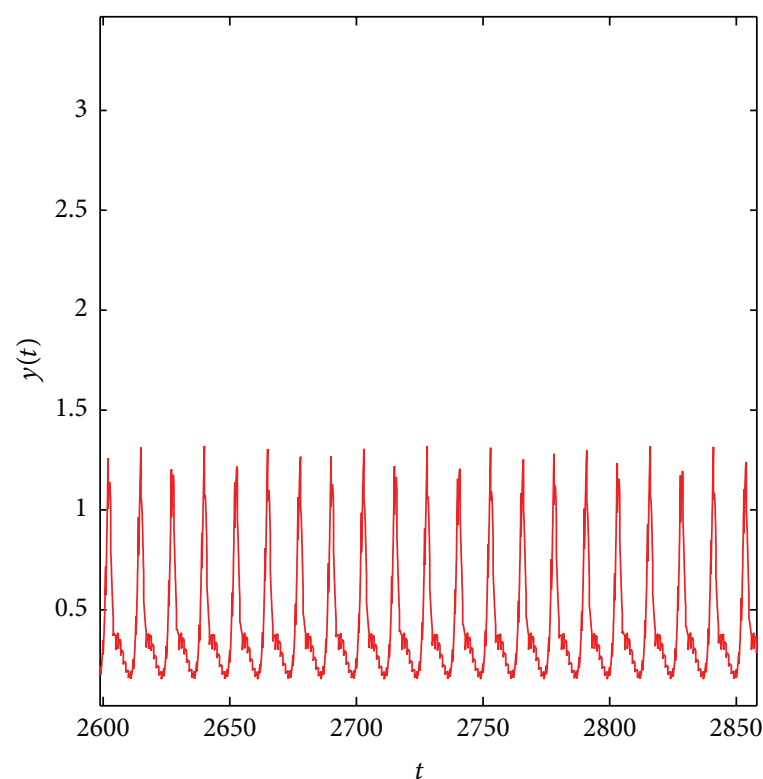

(b)

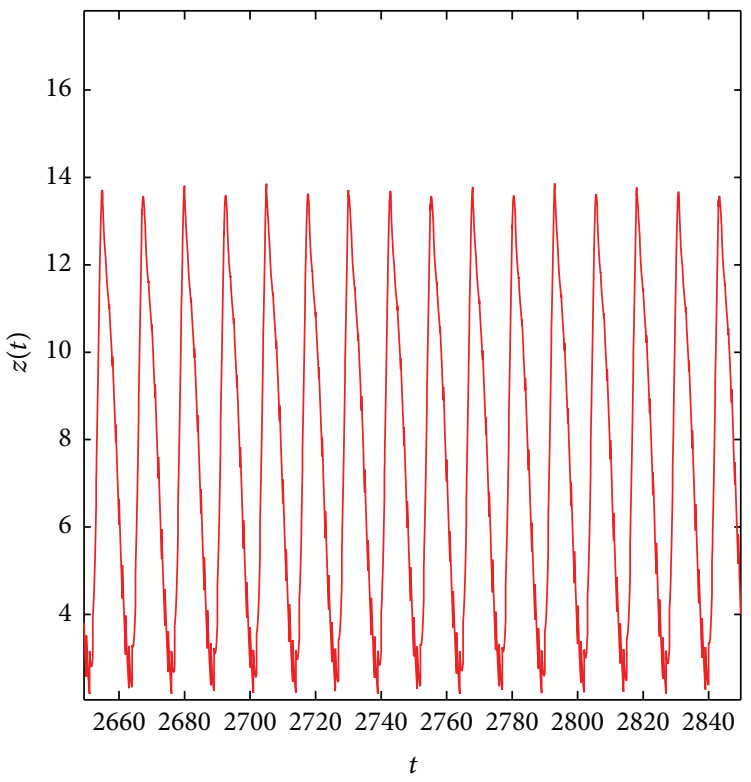

(c)

Figure 8: (a)-(c) Time series of pests $x(t)$ and $y(t)$ depicting decreased biomass level while time series of predator $z(t)$ exhibits increased biomass level of system (2) with mixed Holling type III functional responses in presence of seasonal perturbation with data set (45) and $P=1.2$.

Theorem 14. The periodic solution $\left(0,0, z^{*}(t)\right)$ of system (3) with mixed Holling type III-IV functional responses is locally asymptotically stable if

$$
\begin{aligned}
& T<\frac{1}{a_{1}\left(1+\varepsilon_{1}\right)} \ln \left(\frac{1}{\left(1-\delta_{1}\right)}\right), \\
& \frac{a_{2}\left(1+\varepsilon_{2}\right) T}{c_{2}}-\frac{P\left[(1-A)+\left(1-\delta_{3}\right)(A-B)\right]}{d_{1}\left(1+\varepsilon_{3}\right)\left(1-\left(1-\delta_{3}\right) B\right)}
\end{aligned}
$$

$$
<\frac{1}{c_{2}} \ln \left(\frac{1}{\left(1-\delta_{2}\right)}\right) \text {, }
$$

where $A=\exp \left(-d_{1}\left(1+\varepsilon_{3}\right) l T\right), B=\exp \left(-d_{1}\left(1+\varepsilon_{3}\right) T\right)$.

Remark 15. If we consider the condition $\sin (\omega t)=\sin (\omega t+$ $\psi)=-1$ (see [31]), then similar results can be obtained by the above procedure by replacing $\varepsilon_{i}$ with $-\varepsilon_{i}(i=1,2,3)$. 


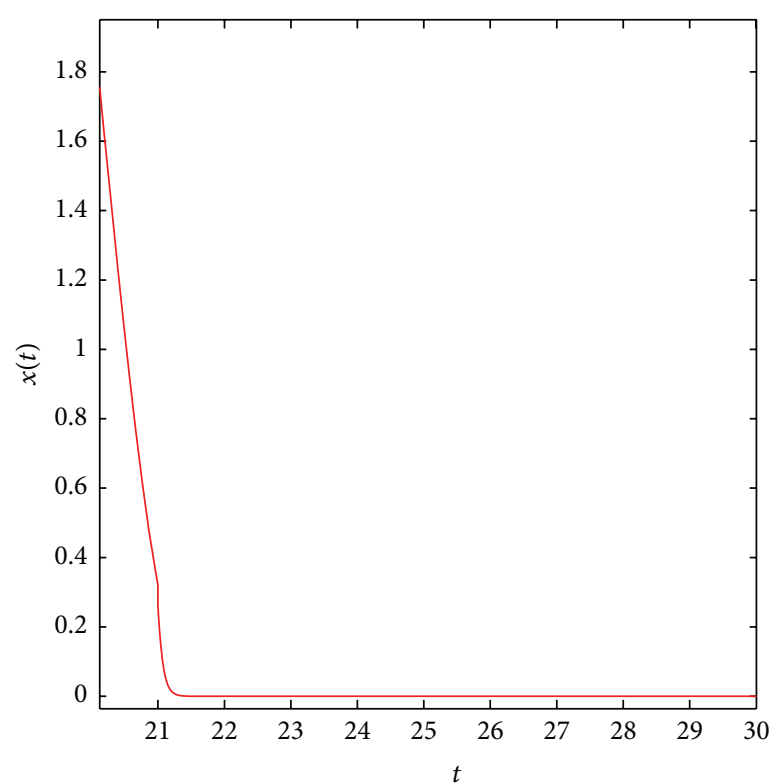

(a)

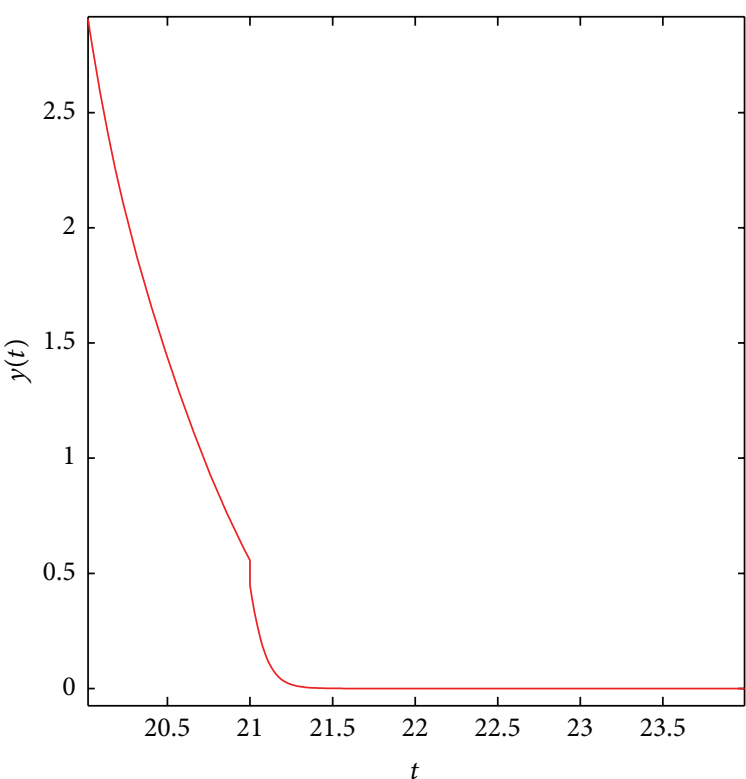

(b)

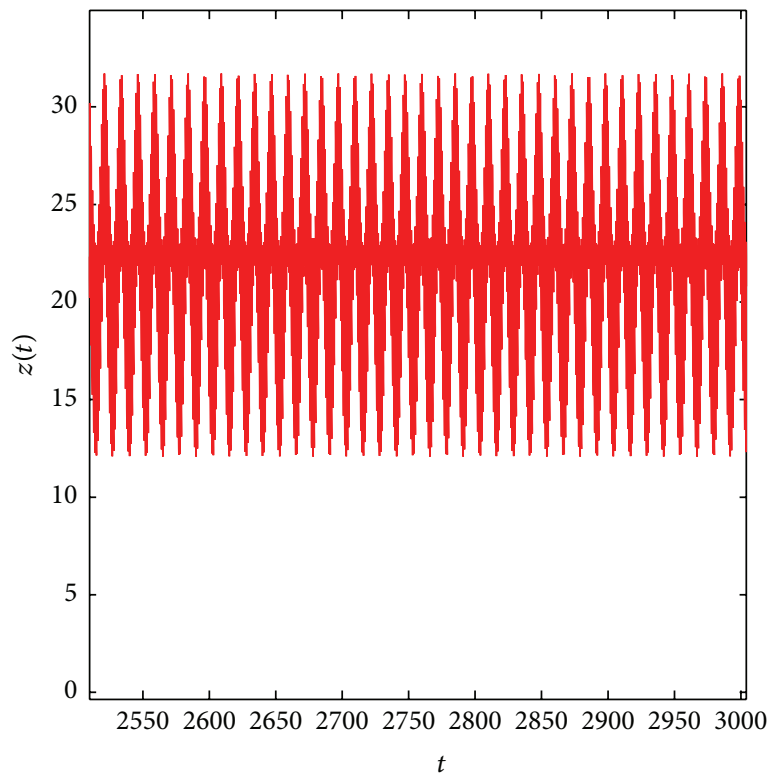

(c)

Figure 9: ((a) and (b)) Time series showing extinction of pests $x(t)$ and $y(t)$ populations; (c) time series showing high biomass density of $z(t)$ population of system (2) with mixed Holling type II functional responses $(p=1, q=1, s=1$, and $r=1)$ in presence of seasonal perturbation with data set (45) and relatively high $P=12$.

\section{Numerical Simulation and Results}

In this section we numerically simulate systems (1) and (2) to investigate the changing dynamics of the systems due to seasonal variation and impulsive perturbation using Matlab R2012a. Firstly, the effects of seasonality in the system will be studied without impulsive perturbation. Then the combined influences of seasonal disturbances and impulsive perturbation on system (2) are discussed. The biologically feasible parametric values are given below:

$$
\begin{aligned}
& a_{1}=3.5, \\
& a_{2}=3.1,
\end{aligned}
$$

$$
\begin{aligned}
e_{1} & =1.0, \\
e_{2} & =1.0, \\
c_{1} & =1.0, \\
c_{2} & =0.9, \\
m_{1} & =0.9, \\
m_{2} & =0.8, \\
d_{1} & =0.5, \\
\omega & =0.4,
\end{aligned}
$$




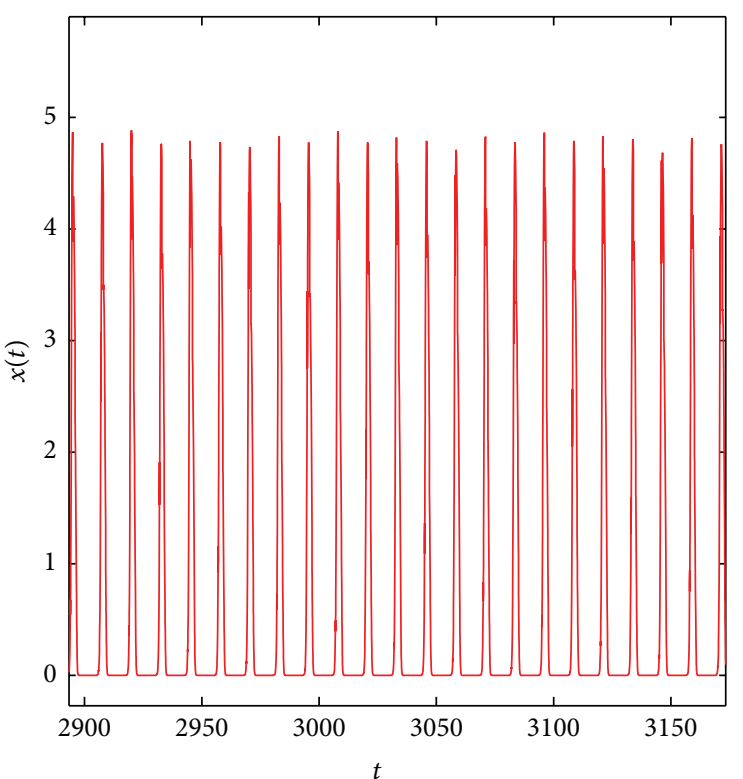

(a)

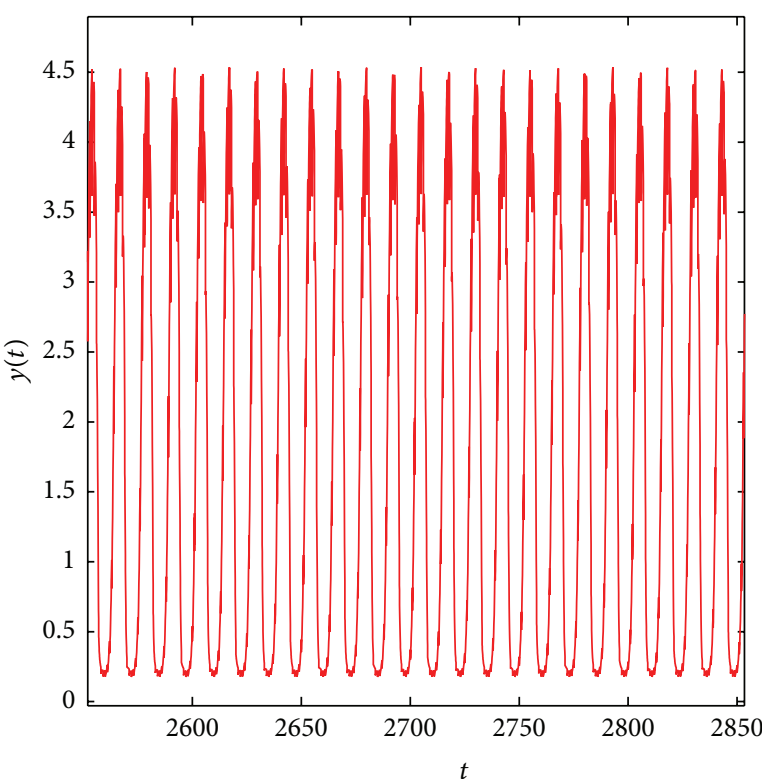

(b)

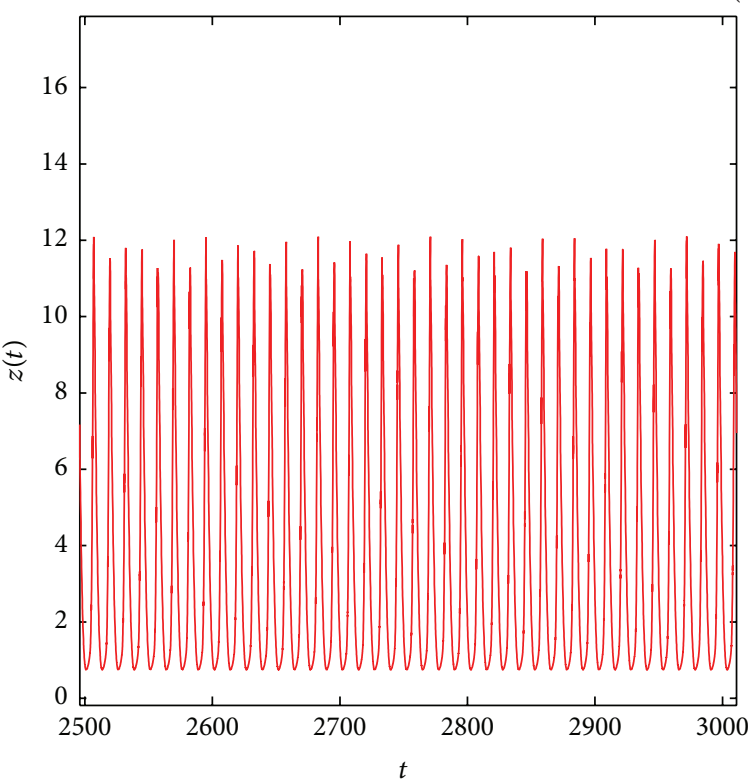

(c)

FIgURE 10: ((a) and (b)) Time series showing increased biomass densities $x(t)$ and $y(t)$ pest populations; (c) time series showing low biomass level of $z(t)$ population of system (2) with mixed Holling type II-III functional responses $(p=1, q=1, s=2$, and $r=2)$ in presence of seasonal perturbation with data set (45) and $P=0.1$.

$$
\begin{aligned}
\varepsilon_{1} & =0.8, \\
\varepsilon_{2} & =0.6, \\
\varepsilon_{3} & =0.35, \\
\psi & =3.142, \\
\phi & =1.57, \\
\delta_{1} & =0.3, \\
\delta_{2} & =0.2, \\
\delta_{3} & =0.1 .
\end{aligned}
$$

The initial conditions are $((x(0), y(0), z(0))=(0.2,0.3,0.2))$.
The degree of seasonality $\varepsilon_{i}(i=1,2,3)$, angular frequency $\omega$ of the fluctuations caused by seasonality, and difference in phase angle $\psi$ are varied to find the effect of seasonal disturbances on system (1). Also the impulsive parameter $P$ is gradually increased to observe the effect of periodic impulsive release of $z(t)$ population in system (2).

6.1. Effect of Seasonal Disturbances on the Model without Impulse. From the numerical simulation we find that Holling type III, type IV, mixed type II-III, and mixed type III-IV systems are initially stable but exhibit periodic oscillations in presence of seasonal variations, whereas Holling type II and 


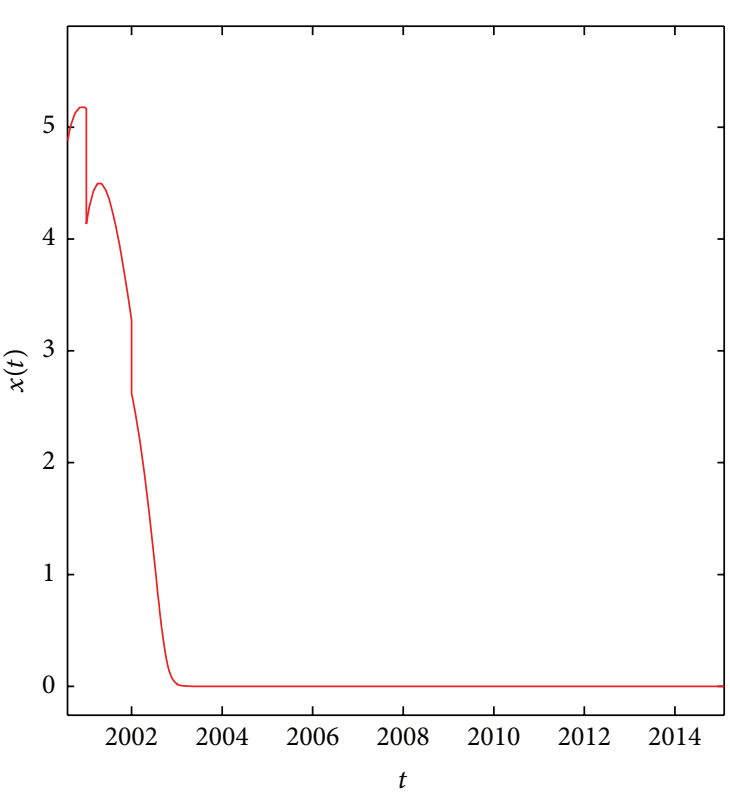

(a)

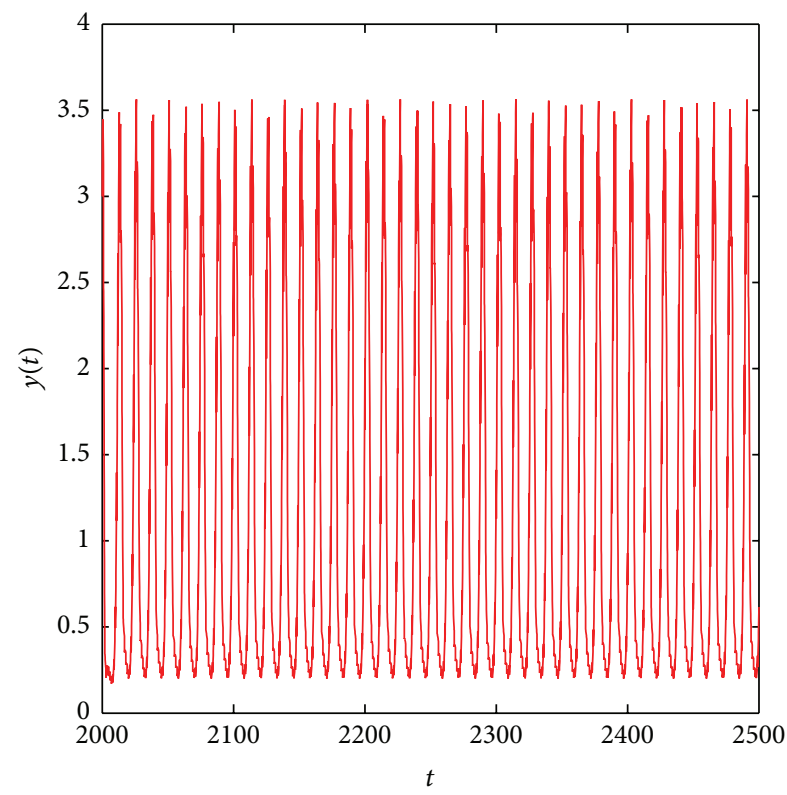

(b)

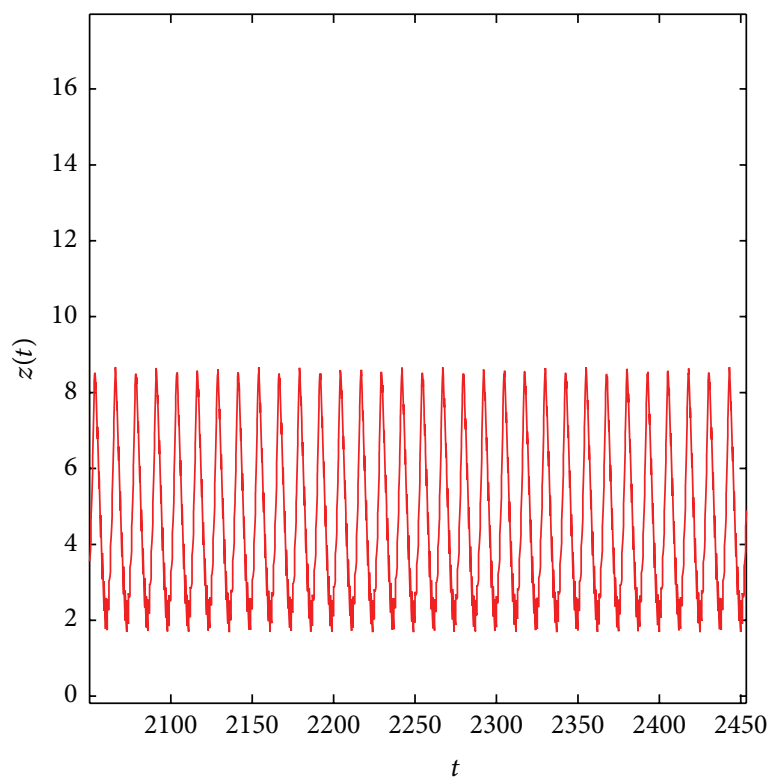

(c)

FIGURE 11: (a) Time series showing decay of $x(t)$ population, (b) time series showing relatively high biomass density of $y(t)$ population, and (c) time series showing low biomass density of $z(t)$ population of system (2) with mixed Holling type II-III functional responses $(p=1$, $q=1, s=2$, and $r=2$ ) in presence of seasonal perturbation with data set (45) and $P=1.2$.

mixed type II-IV system dynamics change from limit cycle to period two and quasiperiod oscillations, respectively.

Small and large amplitude fluctuations can be found depending upon the intensity of the disturbances (mainly degree of seasonality and difference in phase angles between the seasonal parameters). It is observed that biomass level of the species increases significantly in presence of seasonality. It is further observed that there is no possibility of extinction and all populations can coexist in seasonally varying environment. The population time series shows the asymptotic stable behavior for Holling type III system in absence of seasonality (see Figure 1(a)). The solution trajectory exhibits oscillatory behavior in presence of seasonal disturbances (see Figure 1(b)). The population time series shows periodic behavior for mixed Holling type II-IV system in absence of seasonal variations (see Figure 2(a)) while quasiperiodic behavior is found in presence of seasonal variations (see Figure 2(b)).

$x(t)$ and $y(t)$ show in-phase, outer phase, and antiphase synchronous oscillation (see Figure 3 ) for different values of $\psi$. The effect of changing angular frequency $\omega$ in the dynamics of the system has been analyzed. Figure 4 depicts the local 


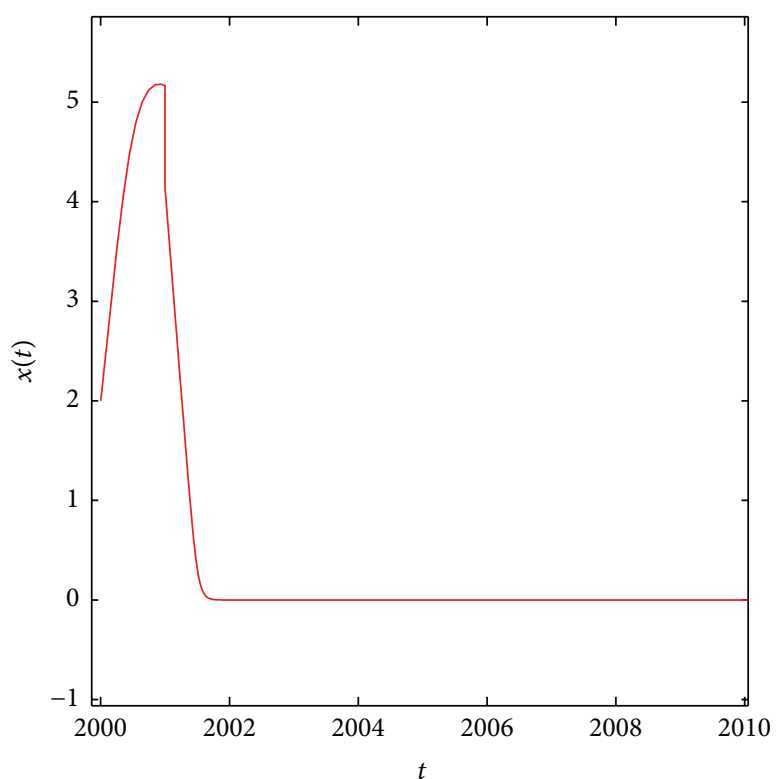

(a)

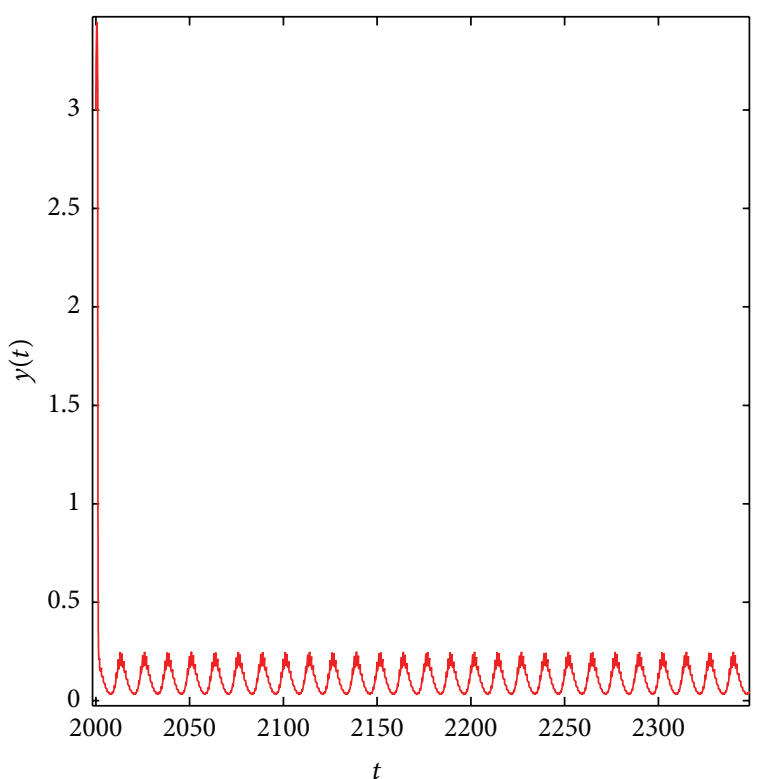

(b)

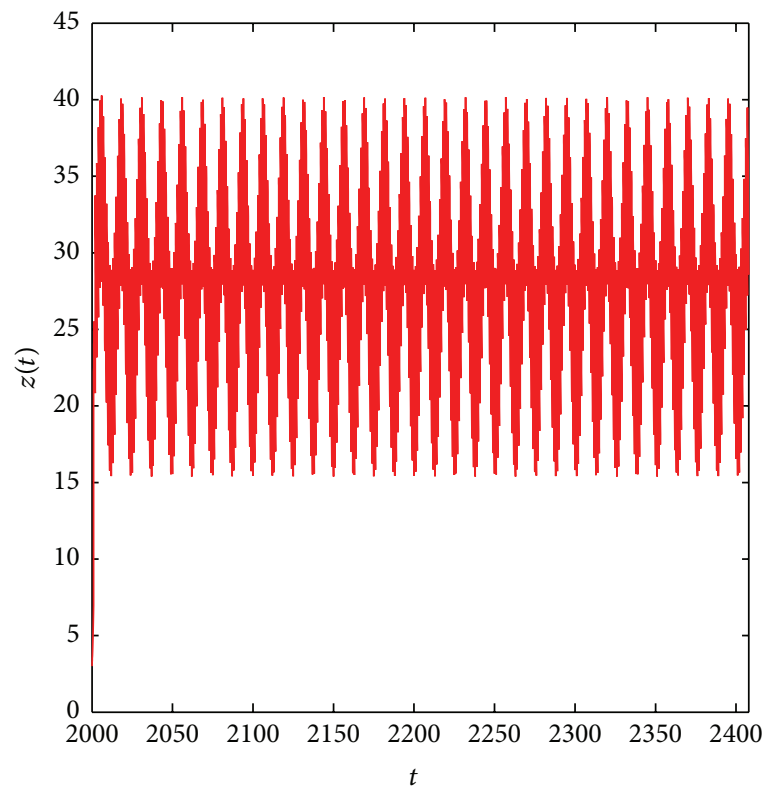

(c)

FIGURE 12: ((a) and (b)) Time series showing extinction of $x(t)$ population, time series showing increased biomass level of $y(t)$ population; (c) time series showing relatively low biomass level of $z(t)$ population of system (2) with mixed Holling type II-III functional responses ( $p=1$, $q=1, s=2$, and $r=2$ ) in presence of seasonal perturbation with data set (45) and $P=12$.

long term dynamics for $\psi=0, \pi / 2, \pi$ when $\omega$ varies from 0 to 0.4 . If $\omega$ increases from 0 to 0.09 , the system has rich changes in dynamics and shows periodic windows, perioddoubling, chaos, and a chaotic crisis (see Figure 4(a)). Figures 4(b) and 4(c) show same pattern when $0<\omega<0.14$ and $0<\omega<0.2$, respectively. These figures show that the size of the chaotic attractor abruptly changes, exhibiting attractor crisis. Hence, it is observed that the seasonality has a strong effect on the dynamical behaviors of system (1). The bifurcation diagrams of system (1) are plotted involving $x(t)$, $y(t)$, and $z(t)$ populations with respect to different seasonal parameters like $\psi$ and $\varepsilon_{i}(i=1,2,3)$. The resulting bifurcation diagrams clearly show that system (1) has rich dynamics including periodic oscillation, period-doubling bifurcation, period-halving bifurcation, and chaos (see Figures 5 and 6).

6.2. Combined Effect of Impulsive and Seasonal Perturbations. Here we examine how the period of release natural enemies affects the dynamical behavior of system. In general, it is found that when the values of $\varepsilon_{i}$ are relatively high and $P$ is in low range $0.1<P<0.9, x(t)$ and $y(t)$ (pests) population biomass grow whereas $z(t)$ (natural enemy or predator) biomass significantly declines (see Figure 7). For moderate value of $P(1<P<1.9), y(t)$ population (second pest) 


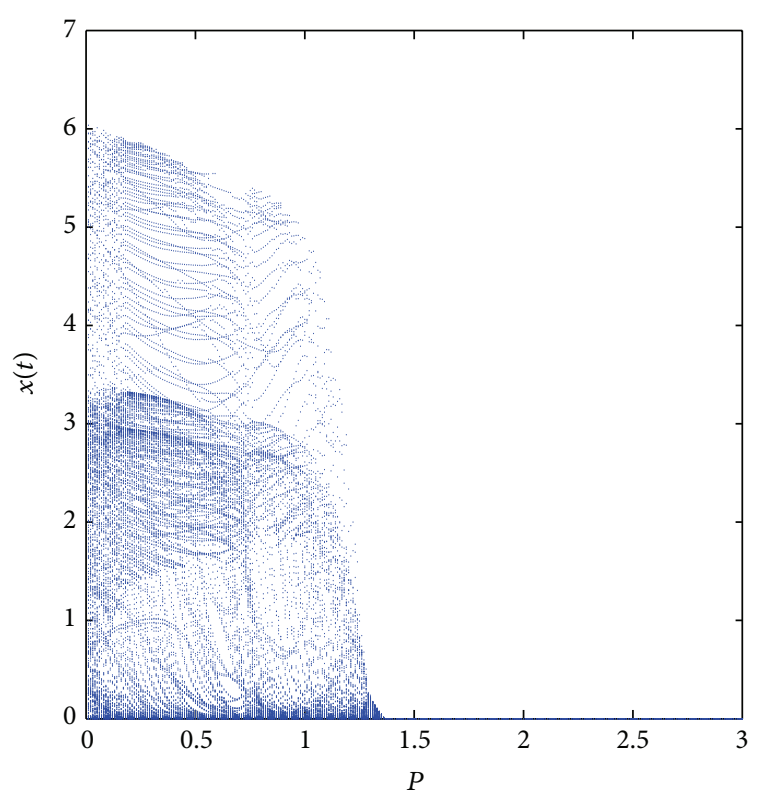

(a)

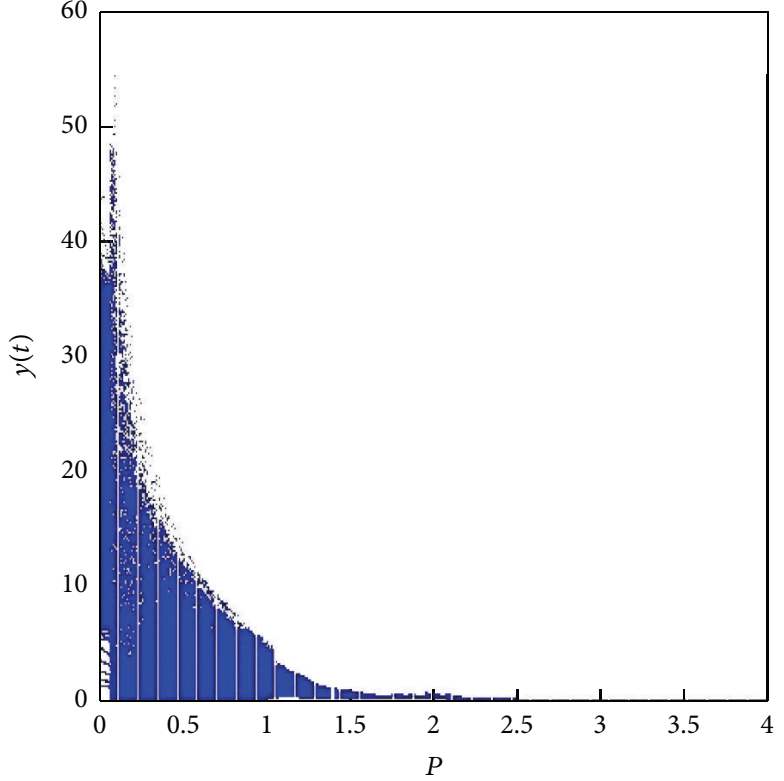

(b)

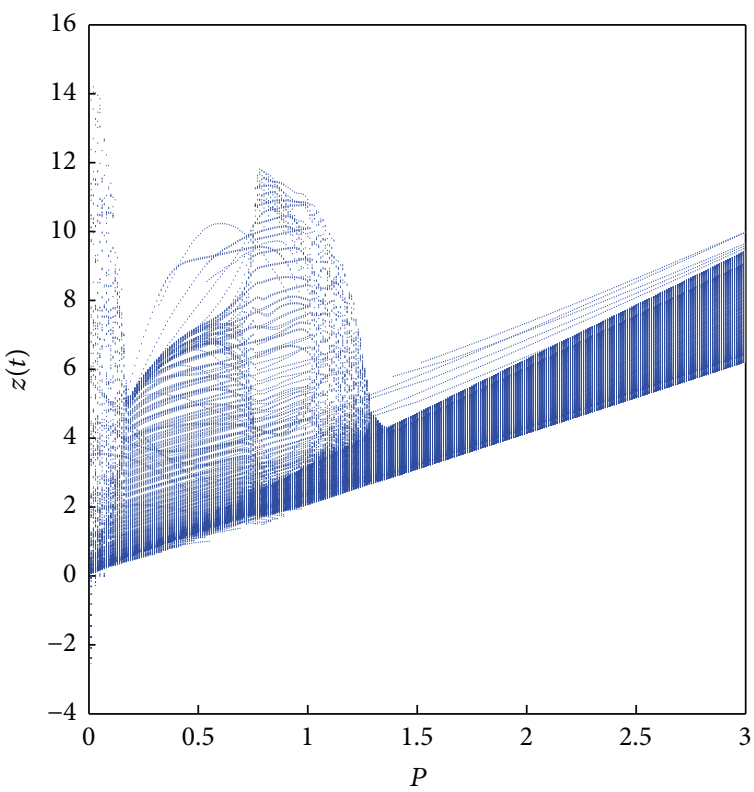

(c)

FIGURE 13: Bifurcation diagram of system (2) with mixed Holling type II functional responses ( $p=1, q=1, s=1$, and $r=1$ ) (a) with respect to $x(t)$, (b) with respect to $y(t)$, and (c) with respect to $z(t)$, in presence of seasonal perturbation where $P$ varies from 0.01 to 3 with data set (45) and $T=1$.

biomass attains lower values and $z(t)$ population biomass does not increase significantly while $x(t)$ population (first pest) increases relatively to a larger value (see Figure 8). But for excessive high values of $P(P>1.9), x(t)$ and $y(t)$ population biomass go to extinction. It is also observed that $z(t)$ biomass density increases up to a level and then fluctuates within a certain range even if the value of $P$ is increased further (see Figure 9).

Mixed Holling type II-III system shows a slightly different result as compared to other Holling type systems. Initially three species are densely populated for $P \in(0.1,0.9)$ (see Figure 10) but for increasing values of $P(1<P<1.9) x(t)$ population goes into extinction, $y(t)$ population biomass fluctuates, and $z(t)$ biomass density increases (see Figure 11). For $P>1.9, y(t)$ population biomass shows an decreasing trend while $x(t)$ population becomes extinct and $z(t)$ population biomass increases (see Figure 12). The bifurcation diagrams with respect to $P$ illustrates quasiperiodic, periodic oscillation and stable behavior when $P$ varies from 0.01 to 3 . (see Figure 13). 


\section{Discussions}

In this paper the seasonally perturbed two prey and one predator with general Holling type functional responses have been investigated. The condition for uniform persistence of system (1) has been derived. It is observed that the seasonal variation plays vital role in the system dynamics in the following manner:

(i) it has significant effect in changing dynamical complexity like periodic, quasiperiodic oscillation;

(ii) it increases the level of biomass concentration of populations in system (1);

(iii) the numerical results indicate that the seasonality with and without phase differences can give rise to complex dynamics including multiple attractors and chaos.

The effect of impulsive perturbation on dynamics of the system is analyzed with significant findings. The impulsive control acts periodically. Using Floquet theory and small amplitude perturbation method the conditions for extinction of the prey (pest) populations are derived under certain parametric restrictions. If $x(t)$ and $y(t)$ are considered as target pest and nontarget pest (harmless pest), respectively, and $z(t)$ is considered as natural enemy for biological control, target pest can be eradicated. For suitable impulsive period $1<P<1.9$ the target pest is eliminated and the nontarget pest (or harmless pest) is prevented from extinction. If both the prey are assumed to be target pests, the impulsive release must be greater than 1.9 for driving them into extinction.

Our results suggest a new approach in biological control of the pest. For example, ladybugs and in particular their larvae are active between May and July in the northern hemisphere. They are voracious predators of aphids and will also consume mites, scale insects, and small caterpillars. The present model can be designed as mathematical model for nontarget pest (small caterpillars), target pest (aphids), natural enemy of the pest, or beneficial predator (ladybugs). Impulsive control method can be used to control pests if the biomass of the natural enemy or predator can always be kept at a certain level so that the volume of pest biomass cannot reach an alarming level. Parasitoid reduces aphids biomass by constantly attacking them and subsequently saving the garden from its attack.

\section{Competing Interests}

The authors declare that they have no competing interests.

\section{References}

[1] C. S. Holling, "The components of predation as revealed by a study of small-mammal predation of the european pine sawfly," The Canadian Entomologist, vol. 91, no. 5, pp. 293-320, 1959.

[2] C. S. Holling, "The functional response of predator to prey density and its role in mimicry and population regulation," Memoirs of the Entomological Society of Canada, vol. 97, no. 45, pp. 1-60, 1965.
[3] J. F. Andrews, "A mathematical model for the continuous culture of microorganisms utilizing inhibitory substrates," Biotechnology and Bioengineering, vol. 10, no. 6, pp. 707-723, 1968.

[4] P. Y. Pang and M. Wang, "Non-constant positive steady states of a predator-prey system with non-monotonic functional response and diffusion," Proceedings of the London Mathematical Society, vol. 88, no. 1, pp. 135-157, 2004.

[5] W. Sokol and J. Howell, "Kinetics of phenol oxidation by washed cells," Biotechnology and Bioengineering, vol. 23, no. 9, pp. 20392049, 1981.

[6] S. G. Ruan and D. G. Xiao, "Global analysis in a predatorprey System with Nonmonotonic functional response," SIAM Journal on Applied Mathematics, vol. 61, no. 4, pp. 1445-1472, 2001.

[7] J. S. Tener, Muskoxen, Queens Printer, Ottawa, Canada, 1965.

[8] R. E. Kooij and A. Zegeling, "Qualitative properties of twodimensional predator-prey systems," Nonlinear Analysis: Theory, Methods \& Applications, vol. 29, no. 6, pp. 693-715, 1997.

[9] J. Sugie, R. Kohno, and R. Miyazaki, "On a predator-prey system of Holling type," Proceedings of the American Mathematical Society, vol. 125, no. 7, pp. 2041-2050, 1997.

[10] N. D. Kazarinoff and P. van den Driessche, "A model predatorprey system with functional response," Mathematical Biosciences, vol. 39, no. 1-2, pp. 125-134, 1978.

[11] A. Gragnani and S. Rinaldi, "A universal bifurcation diagram for seasonally perturbed predator-prey models," Bulletin of Mathematical Biology, vol. 57, no. 5, pp. 701-712, 1995.

[12] A. R. Ives, K. Gross, A. Vincent, and A. Jansen, "Periodic mortality events in predator-prey systems," Ecology, vol. 81, no. 12, pp. 3330-3340, 2000.

[13] S. Rinaldi, S. Muratori, and Y. Kuznetsov, "Multiple attractors, catastrophes and chaos in seasonally perturbed predator-prey communities," Bulletin of Mathematical Biology, vol. 55, no. 1, pp. 15-35, 1993.

[14] R. A. Taylor, A. White, and J. A. Sherratt, "How do variations in seasonality affect population cycles?" Proceedings of the Royal Society B: Biological Sciences, vol. 280, no. 1754, 2013.

[15] K. Saleh, "Multiple attractors involving chaos in predatorprey model with non monotonic response function response," International Journal of Basic and Applied Sciences, vol. 11, no. 4, pp. 38-48, 2011.

[16] B. Sahoo, "Predator-prey system with seasonally varying additional food to predators," International Journal of Basic and Applied Sciences, vol. 1, no. 4, pp. 363-373, 2012.

[17] G. C. W. Sabin and D. Summers, "Chaos in a periodically forced predator-prey ecosystem model," Mathematical Biosciences, vol. 113, no. 1, pp. 91-113, 1993.

[18] S. Gakkhar and R. K. Naji, "Chaos in seasonally perturbed ratiodependent prey-predator system," Chaos, Solitons and Fractals, vol. 15, no. 1, pp. 107-118, 2003.

[19] H. Yu and M. Zhao, "Seasonally perturbed prey-predator ecological system with the Beddington-DeAngelis functional response," Discrete Dynamics in Nature and Society, vol. 2012, Article ID 150359, 12 pages, 2012.

[20] Y. Pei, G. Zeng, and L. Chen, "Species extinction and permance in a prey-predator model with two-type functional responses and impulsive biological control," Nonlinear Dynamics, vol. 52, no. 1, pp. 71-81, 2008.

[21] X. Wang, W. Wang, and X. Lin, "Dynamics of a two-prey one-predator system with Watt-type functional response and impulsive control strategy," Chaos, Solitons and Fractals, vol. 40, no. 5, pp. 2392-2404, 2009. 
[22] Y. Zhang, Z. Xiu, and L. Chen, "Dynamic complexity of a twoprey one-predator system with impulsive effect," Chaos, Solitons and Fractals, vol. 26, no. 1, pp. 131-139, 2005.

[23] X. Song and Y. Li, "Dynamic complexities of a Holling II twoprey one-predator system with impulsive effect," Chaos, Solitons \& Fractals, vol. 33, no. 2, pp. 463-478, 2007.

[24] H. Liu and F. Meng, "Existence of positive periodic solutions for a predator-prey system of Holling type IV function response with mutual interference and impulsive effects," Discrete Dynamics in Nature and Society, vol. 2015, Article ID 138984, 12 pages, 2015.

[25] H. Li, L. Zhang, Z. Teng, and Y. Jiang, "Dynamic behaviors of Holling type II predator-prey system with mutual interference and impulses," Discrete Dynamics in Nature and Society, vol. 2014, Article ID 793761, 13 pages, 2014.

[26] Z. Wang, Y. Shao, X. Fang, and X. Ma, "An impulsive threespecies model with square root functional response and mutual interference of predator," Discrete Dynamics in Nature and Society, vol. 2016, Article ID 3897234, 14 pages, 2016.

[27] H. Baek, "Species extinction and permanence of an impulsively controlled two-prey one-predator system with seasonal effects," BioSystems, vol. 98, no. 1, pp. 7-18, 2009.

[28] H. Baek, "An impulsive two-prey one-predator system with seasonal effects," Discrete Dynamics in Nature and Society, vol. 2009, Article ID 793732, 19 pages, 2009.

[29] H. Yu, S. Zhong, R. P. Agarwal, and S. K. Sen, "Effect of seasonality on the dynamical behavior of an ecological system with impulsive control strategy," Journal of the Franklin Institute, vol. 348, no. 4, pp. 652-670, 2011.

[30] X. Ma, Y. Shao, Z. Wang, X. Fang, and Z. Luo, "Analysis of an impulsive one-predator and two-prey system with stage-structure and generalized functional response," Discrete Dynamics in Nature and Society, vol. 2015, Article ID 864367, 10 pages, 2015.

[31] C. Dai, M. Zhao, and L. Chen, "Complex dynamic behavior of three-species ecological model with impulse perturbations and seasonal disturbances," Mathematics and Computers in Simulation, vol. 84, pp. 83-97, 2012.

[32] F. D. Chen, "On a nonlinear nonautonomous predator-prey model with diffusion and distributed delay," Journal of Computational and Applied Mathematics, vol. 180, no. 1, pp. 33-49, 2005.

[33] V. Lakshmikantham, D. D. Bainov, and P. S. Simeonov, Theory of Impulsive Differential Equations, vol. 6 of Series in Modern Applied Mathematics, World Scientific Publishing, Teaneck, NJ, USA, 1989.

[34] D. Bainov and P. Simeonov, Impulsive Differential Equations: Asymptotic Properties of the Solutions, vol. 28 of Series on Advances in Mathematics for Applied Sciences, World Scientist, River Edge, NJ, USA, 1995.

[35] G. Birkhorff and G. C. Rota, Ordinary Differential Equations, Ginn Press, Needham Heights, Mass, USA, 1982. 


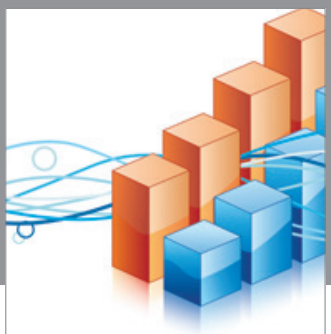

Advances in

Operations Research

vatem alat4

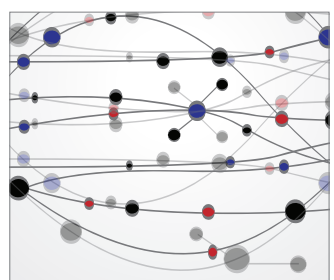

\section{The Scientific} World Journal
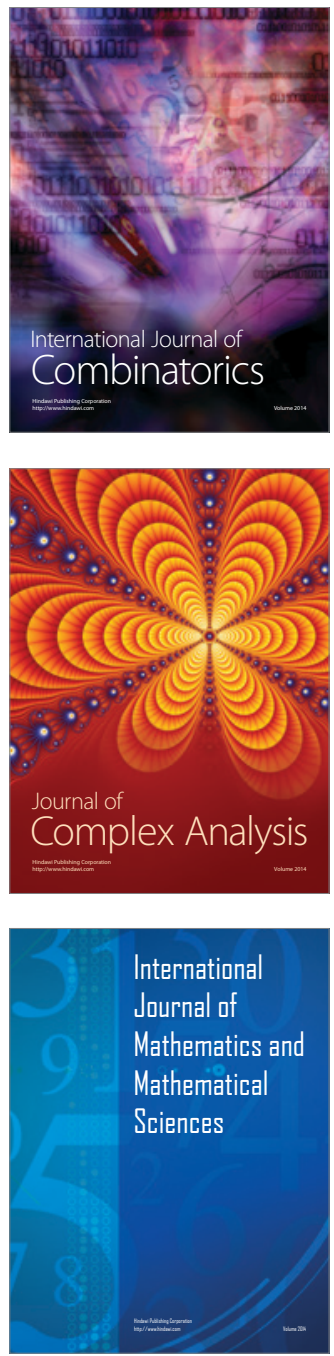
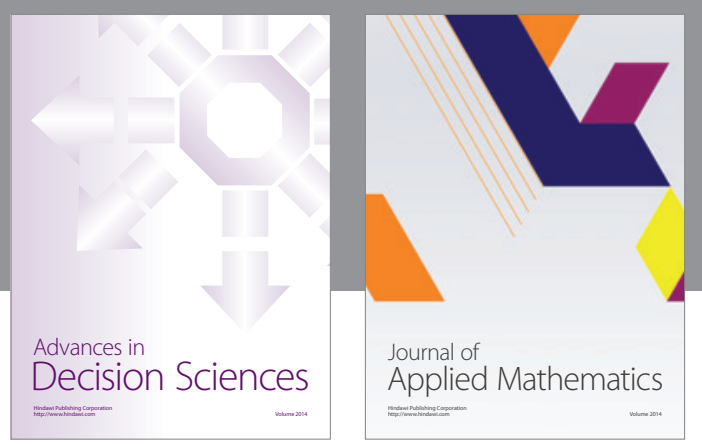

Algebra

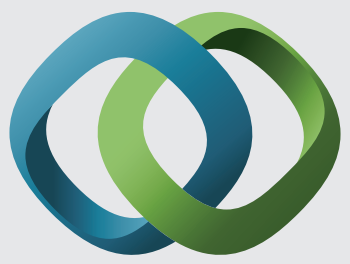

\section{Hindawi}

Submit your manuscripts at

http://www.hindawi.com
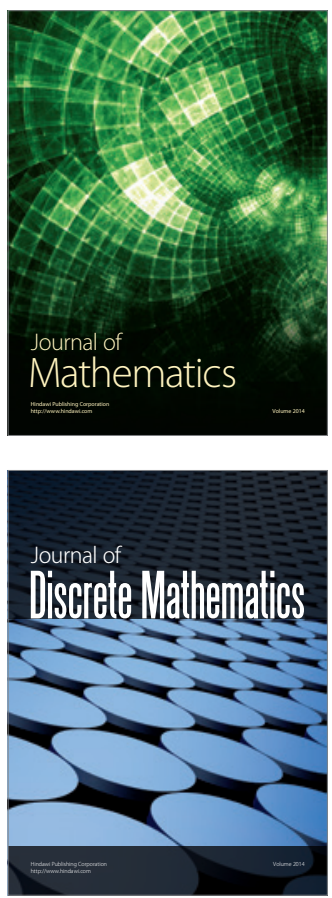

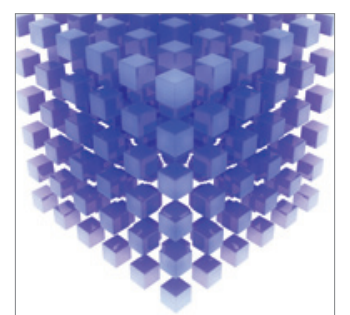

Mathematical Problems in Engineering
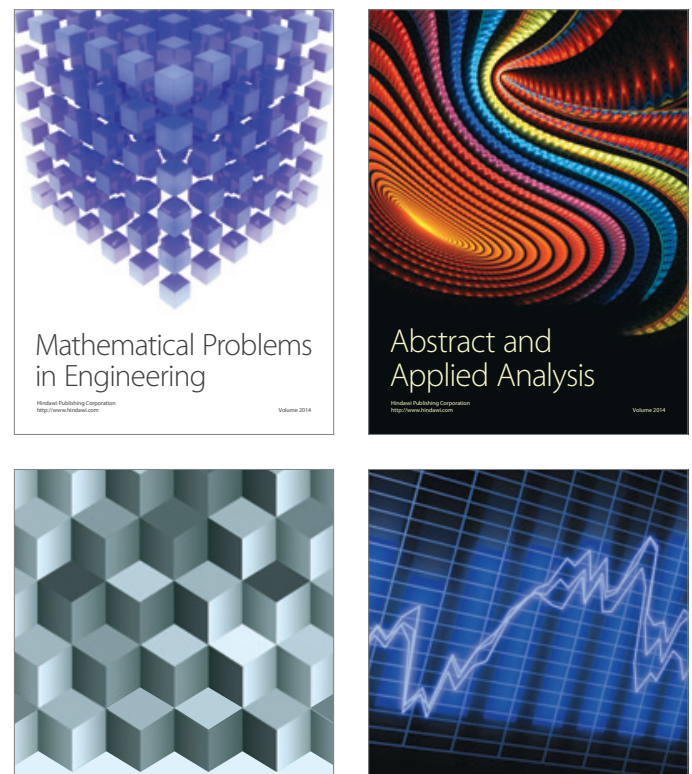

Journal of

Function Spaces

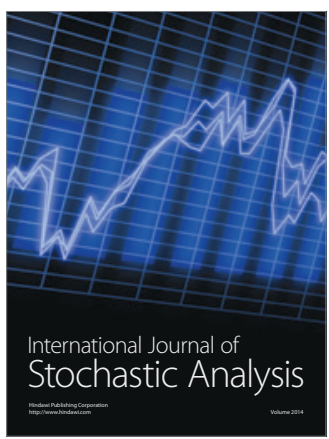

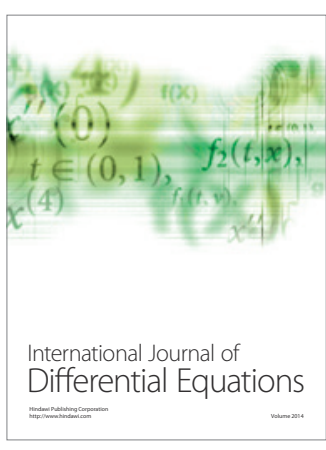
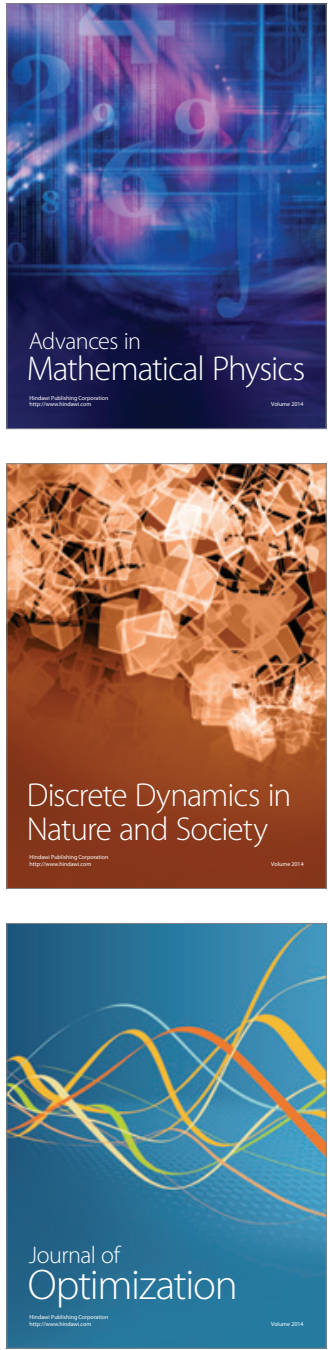